\title{
The stability of immiscible viscous fingering in Hele-Shaw cells with spatially varying permeability
}

\author{
S.J. Jackson, H. Power*, D. Giddings, D. Stevens \\ Fluids and Thermal Engineering Research Group, University of Nottingham, UK
}

\begin{abstract}
In this paper, we investigate the stability of immiscible viscous fingering in Hele-Shaw cells with spatially varying permeability, across a range of capillary numbers. We utilise a coupled boundary element - radial basis function (BE - RBF) numerical method that adapts and moves with the growing interface, providing an efficient, high accuracy scheme to track the interfacial displacement of immiscible fluids. By comparing the interfacial evolution and growth rate in varying permeability cells to that in uniform cells, we can assess the relative stability of the perturbations as a consequence of the variable permeability.

Numerical experiments in Hele-Shaw cells with gradually varying permeability highlight 3 aperture effects that control the interfacial stability: 1) Gradients in the capillary pressure 2) Local changes in fluid mobility 3) Variation in the viscous pressure gradient. In low capillary number regimes, we find that aperture effect 1 and 2 dominate, which (relatively) stabilise interfacial perturbations in converging geometries and destabilise perturbations in diverging geometries. In high capillary number regimes, aperture effect 3 dominates meaning the relative stability transitions; the interface is destabilised in converging cells and stabilised in diverging cells. We find an upper bound critical capillary number $C a_{g t}$ at which the relative stability transitions in our gradually varying cell as $1000<C a_{g t}<1250$, which is independent of both $\alpha$ and $\epsilon_{0}$. This result is much lower than the value of $C a_{g t}=9139$ predicted by linear stability theory, due to significant nonlinear perturbation growth. This transition links the results found in previous works performed at low and high capillary numbers, providing new insight into the viscous fingering instability in variable permeability cells.

To conclude, we present simulations in Hele-Shaw cells with large geometric heterogeneities and anisotropy, in order to demonstrate the significant fluid re-distribution that can occur due to localised variations in cell permeability. Using periodic permeability distributions, we show the significant re-distribution of fluid that can occur due to large capillary pressure gradients in the capillary limit, and the channelling of flow that can occur in the viscous limit along anisotropic features.
\end{abstract}

Keywords: , Spatially varying permeability, Capillary number, Viscous Fingering Instability, BE $-\mathrm{RBF}$

\section{Introduction}

Since the work of [1], there has been extensive research on the viscous fingering problem occurring in Hele-Shaw cells, where the fluid flows between two thinly separated plates. When a fluid of high viscosity is displaced by one of a lower viscosity, interfacial instabilities can evolve forming complex interfacial patterns in which long fingers of the less viscous fluid penetrate the more viscous fluid. The mobility $M$ of a fluid within a Hele-Shaw cell is defined by the cell plate separation $b$ and the viscosity $\mu, M=b^{2} / 12 \mu$ giving rise to an intrinsic permeability $k=b^{2} / 12$ analogous to that in porous media flows. The study of viscous fingering in Hele-Shaw cells is often

\footnotetext{
*Corresponding author

Email address: henry.power@nottingham.ac.uk (H. Power)
} 
used to provide insight into the more complex problem in porous media, whereby effects such as anisotropic permeability and wetting conditions play a significant role in the interface evolution.

One such porous media flow, and the motivation behind the current work is the injection and storage of supercritical $\mathrm{CO}_{2}$ in deep subsurface aquifers (carbon sequestration). When $\mathrm{CO}_{2}$ is injected into a deep saline geological formation suitable for storage, it will displace the resident brine, creating an immiscible two-phase flow regime. The injected $\mathrm{CO}_{2}$ will rise upwards due to buoyancy forces, eventually hitting a low permeability cap rock where a permeability and capillary barrier stops the $\mathrm{CO}_{2}$ from rising out of the aquifer. After this stage, the $\mathrm{CO}_{2}$ will spread laterally, further displacing brine. In this work, we are interested in the lateral spreading of $\mathrm{CO}_{2}$ and brine under the cap rock, for which we use the analogy between Hele-Shaw and porous media flows.

During the immiscible displacement of brine via the injection of supercritical $\mathrm{CO}_{2}$, the flow regime depends greatly on the capillary number at the interface between the fluids [2]. The local capillary number, $C a_{l}$ is defined as $\mu U_{n} / \gamma$, where $\mu$ is the dynamic viscosity of the displaced fluid, $U_{n}$ is the normal interfacial velocity and $\gamma$ is the surface tension. At capillary numbers above roughly $1 \times 10^{-4}$ with a mobility ratio greater than one, viscous fingering can occur, where long fingers extend into the porous domain from the injection site [3].

A typical subsurface aquifer will have an inhomogeneous permeability that varies in space as a consequence of the way it has been formed and re-shaped over many millions of years, altering the natural stress state of the aquifer [4]. Abrupt changes in aquifer permeability can exist due to fault lines and changes in the aquifer material [4]. As well as pre-existing variations in permeability, the $\mathrm{CO}_{2}$ injection process itself can alter the permeability of the aquifer. During $\mathrm{CO}_{2}$ injection, formation dry-out and precipitation of minerals (such as salt from pre-existing brine) near the injection well can reduce the porosity and permeability of the aquifer [5]. The formation of carbonic acids in the brine due to reactions with the injected $\mathrm{CO}_{2}$ can dissolve the calcite in sandstone aquifers, increasing the permeability [6]. Injection pressures over the formation pressure of the aquifer can also induce fracturing and fault slip, which can increase the permeability in a region surrounding the injection well [7]. Understanding the effect of these permeability variations on the flow regime and interfacial evolution of injected $\mathrm{CO}_{2}$ is critical in understanding the long term storage capabilities of supercritical $\mathrm{CO}_{2}$ and forms the motivation behind the current work.

A spatially varying permeability in a Hele-Shaw cell $\left(k=b^{2} / 12\right)$ is a consequence of the plate separation $b$, which also gives rise to a fluid mobility that varies spatially. Although the viscosity in the fluid mobility could also vary spatially (due to temperature gradients etc.), in this work we analyse the case of an inhomogeneous fluid mobility arising purely from the spatial variation in plate separation and cell permeability.

Recently, several analytical and experimental works have shown that small changes in the plate separation (and hence permeability) in a Hele-Shaw cell can have significant effects on the interface evolution, allowing the control and suppression of fingering instabilities [8]. Using a rectilinear Hele-Shaw cell set-up, [9] investigate secondary tip-splitting instabilities occurring under high capillary number regimes when the cell has a small gradient in plate separation in the flow direction. Experiments show that a small positive gradient flattens the finger tip, making it easier for the finger to bifurcate. In the converging cell case, the finger sharpens, and remains stable for a much longer period compared to the flat plate configuration. Physically, with an almost constant curvature around the finger front in the rectilinear set-up, the finger tip is accelerated most strongly by the converging geometry, creating a sharper tip. The analytical stability analysis of [9] reveals a large dependence of the growth rate of instabilities with the magnitude of the plate separation, but no dependence on the sign of the separation gradient, contrary to experimental results.

[10] use a similar stability analysis to [9] but employ mode coupling theory to provide analysis into the slightly non-linear regime of finger tip splitting in a rectilinear cell. They find dependence on the sign of the plate separation gradient on the finger tip growth in contrast to [9]. Their findings agree with the experimental findings published by [9] for the case of high capillary numbers in the weakly non-linear stage of finger growth.

In contrast to [9] and [10], [11] present results in a rectilinear cell with a gradually varying plate separation under much smaller capillary number regimes, looking at the onset and subsequent control of the fingering instability. In very low capillary number flows, the gradually converging cell can completely stabilise the flow due to the variation in transverse curvature around the interface. 
Areas of the interface displaced further into the cell have a larger transverse curvature, resulting in a larger capillary pressure and stabilising action from the surface tension. The large capillary pressure helps to stabilise the front and can produce a completely flat interface if the capillary number is below a critical value. [11] predict a critical capillary number through experimental testing and analytically using an equation derived from a linear stability analysis (LSA) of the governing Darcy flow regime. This analysis gives a critical capillary number under which perturbations of any wavelength can be stabilised, depending on the viscosity contrast, contact angle and plate gradient. The analytical predictions agree remarkably well with the experimental findings, with small discrepancies likely due to dynamic wetting effects [11].

Utilising a radial Hele-Shaw cell set-up, [12] provides early insight into the case of a slightly diverging cell, showing that viscous fingering is initially delayed. This was later confirmed analytically by [8], who show that the radial growth of a finger has an offset given by $-\alpha r_{0} / h_{0}$, where $\alpha$ is the gap gradient, $r_{0}$ is the interface position and $h_{0}$ is the plate separation respectively. This offset is negative in the diverging geometry causing the interface to be slowed and viscous fingering delayed. [8] also analyse how the radial set-up is affected by converging geometry, showing a similar capillary number limit for which viscous fingering can be completely suppressed.

Another strategy to suppress the fingering instability is the use of a Hele-Shaw cell with an elastic upper membrane, replacing the classic rigid plate. The elastic membrane displaces vertically upon the injection of the less viscous fluid, creating a profile that converges to a uniform plate separation far from the injection site. The expansion of the elastic upper membrane occurs at a rate much faster than the displacement of the outer fluid, meaning the viscous fingering stability can be modelled in a similar vein to the radial case presented by [8], i.e. in a static manner. [13] and [14] show that the converging geometry caused by the rapid cell expansion helps to stabilise the interface between the fluids. This is also demonstrated experimentally by [15], where the interface can be completely stabilised using an elastic membrane for short time periods under low capillary number regimes. At higher capillary numbers, the interface proceeds to form short stubby fingers, also identified by the numerical model in [14].

These theoretical and experimental findings confirm that the evolution of viscous fingering has a large dependence on the plate separation variation (and hence cell permeability) in both rectilinear and radial Hele-Shaw flows. Although a significant amount of previous work exists on the linear growth and early stages of the tip splitting instability with a variable plate separation, analysis of the long term non-linear evolution is lacking. Research has focused on control of the fingering instability, where linear stability theory can be effectively used to analyse the growth rate and finger morphology if the instabilities are suppressed early in time. However, to fully understand the nonlinear finger interactions and late stage interfacial evolution in radial Hele-Shaw flow subject to a variable plate separation requires extensive numerical simulation [10].

The contrasting results at low and high capillary numbers in rectilinear cells mentioned previously (in converging cells, fingers are smoothed at low capillary numbers and sharpened at high capillary numbers) indicate a transition in fingering mechanisms with capillary number. This transition has not been explored in detail, with previous authors generally focusing on one region due to the application of their study. However, in the present work the transition region is explicitly explored, in order to provide detail on the regimes that could occur under the flow regimes present in $\mathrm{CO}_{2}$ sequestration. Here, the capillary number is initially high, but decreases with time, indicating that both high and low capillary number regimes may be experienced by the advancing plume.

By varying the capillary number in a simple uniformly converging/diverging cell, we can systematically explore the transition in effects that have been alluded to in the results of previous papers. The numerical method allows analysis at much later time-scales and in greater detail than has previously been attempted. After these initial experiments, we present simulations in HeleShaw cells with much larger heterogeneities. We demonstrate the significant fluid re-distribution that can occur in more complex geometries when 1) the capillary number is low and gradients in the capillary pressure (due to the plate separation) control the flow regime and 2) when the capillary number is high and anisotropic permeability controls the flow regime. Unlike previous studies, we do not provide a control strategy for the fingering instabilities, instead presenting detailed analysis and discussion on the long term effects of varying cell permeability on the interfacial evolution.

Throughout this work we refer to both plate separation and cell permeability interchangeably, 
since a change in one induces a corresponding change in the other, i.e. $k=b^{2} / 12$. All the conclusions drawn concerning the effects of changing plate separation on the immiscible flow regime are therefore also true when considering the corresponding change in permeability and vice versa.

In the following paper, we start by formulating the mathematical model in $\S 2$ followed by an overview of the numerical methods. We provide numerical verification of the coupled scheme in $\S 4$. In $\S 5$ we present numerical experiments with detailed discussion and comparison with previous work, concluding in final remarks and findings in $\S 6$.

\section{Mathematical model}

We consider a circular Hele-Shaw cell of infinite radius, in which high viscosity fluid is displaced by the injection of a less viscous fluid. The low viscosity invading fluid (such as $\mathrm{CO}_{2}$ ) occupies region $\Omega_{1}$ whilst a high viscosity fluid (such as brine) occupies the external region $\Omega_{2}$ shown in Figure 1. The initial interface has a perturbation given in Figure 1, where $\epsilon_{0}$ and $\theta$ are the perturbation amplitude and the azimuthal angle around the interface. Throughout this article, vectors are written in bold and scalars or components of vectors are written in normal face. The fluid mobility in region $l$ at a point $\boldsymbol{x}=\left(x_{1}, x_{2}\right)$ is related to the Hele-Shaw plate separation $b(\boldsymbol{x})$ and the fluid viscosity $\mu_{l}$ by:

$$
M_{l}(\boldsymbol{x})=\frac{b(\boldsymbol{x})^{2}}{12 \mu_{l}}
$$

The $b(\boldsymbol{x})^{2} / 12$ term in the mobility ratio refers to the intrinsic permeability $k$ of the Hele-Shaw cell, defined by the plate separation. For the flow between two thinly separated plates in a Hele-Shaw cell, the depth averaged pressure $P$ and two dimensional cross-sectional average Darcy velocity $\mathbf{u}$ in each fluid region can be expressed through Darcy's law:

$$
u_{i}^{l}(\boldsymbol{x})=-M_{l}(\boldsymbol{x}) \frac{\partial P_{l}(\boldsymbol{x})}{\partial x_{i}}
$$

The flow field satisfies conservation of mass that can be written in terms of the Darcy velocity and the plate separation as:

$$
\frac{\partial\left(b(\boldsymbol{x}) u_{i}^{l}(\boldsymbol{x})\right)}{\partial x_{i}}=0
$$

Substituting the Darcy velocity (2) into the conservation of mass equation (3):

$$
\frac{\partial}{\partial x_{i}}\left(b(\boldsymbol{x}) M_{l}(\boldsymbol{x}) \frac{\partial P_{l}}{\partial x_{i}}\right)=0 \quad \boldsymbol{x} \in \Omega_{l}, \quad l=1,2
$$

At a boundary point $\boldsymbol{\xi}$ on the fluid interface $S$ between $\Omega_{1}$ and $\Omega_{2}$, there are two matching conditions (kinematic and dynamic) that must be met by the advancing interface. Firstly, the kinematic condition requires the continuity of normal velocity $u_{i}(\boldsymbol{\xi}) n_{i}(\boldsymbol{\xi})$, i.e.

$$
-M_{1}(\boldsymbol{\xi}) \frac{\partial P_{1}(\boldsymbol{\xi})}{\partial n}=-M_{2}(\boldsymbol{\xi}) \frac{\partial P_{2}(\boldsymbol{\xi})}{\partial n}
$$

Secondly, the dynamic condition describes the pressure jump across the interface:

$$
P_{1}(\boldsymbol{\xi})-P_{2}(\boldsymbol{\xi})=\gamma\left(\frac{2}{b(\boldsymbol{\xi})}+\frac{\pi}{4} k(\boldsymbol{\xi})\right)
$$

where $\gamma$ is the surface tension and $k(\boldsymbol{\xi})$ is the curvature. $k(\boldsymbol{\xi})$ is considered a continuous function on the interface surface with a $\pi / 4$ scaling term included for consistency with the asymptotic analysis of [16]. The contact angle of the meniscus has been assumed to be zero and we neglect dynamic wetting effects. Dynamic wetting has been shown by various authors to have a considerable effect on the interfacial displacement in a Hele-Shaw cell at high capillary numbers and requires study in its own right, putting it beyond the scope of the current work [17], [18], [19]. 


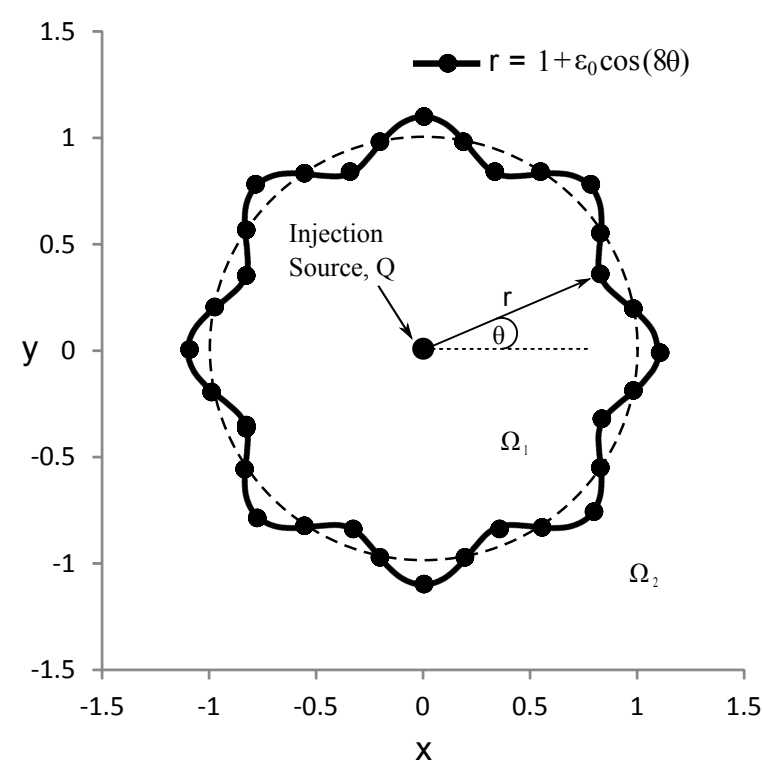

(a)

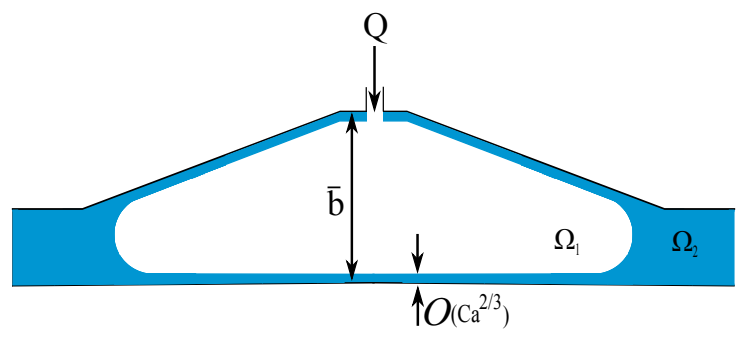

(b)

Figure 1: (a) Planar view of the 2D radial injection problem, with 8 finger symmetric perturbation. (b) Side view of a radially symmetric converging Hele-Shaw cell.

The displacement of the outer fluid is initiated by the injection of the inner fluid with a point source of strength $Q$ at the origin, with velocity given by:

$$
u_{i}=\frac{Q x_{i}}{2 \pi r^{2}}
$$

here, $r$ is the distance from the collocation point, $\boldsymbol{x}$, to the source point located at the origin; i.e. $r=|\boldsymbol{x}|$. The far field pressure tends to that generated by the source at a distance $\boldsymbol{x} \rightarrow \infty$ :

$$
\underset{\boldsymbol{x} \rightarrow \infty}{P_{2}(\boldsymbol{x})} \rightarrow-\frac{Q}{2 \pi M_{2}(\boldsymbol{x})} \ln \left(\frac{r}{r_{0}}\right)
$$

Equation (4), subject to matching conditions (5, 6), and asymptotic condition (8) represents a well posed problem that can be solved with a variety of numerical methods in order to find the pressure in the domain and subsequently the interface velocity. In this work, in order to characterise the specific effects of the varying plate separation in comparison to the uniform cell case (which has been presented previously by [20], the variables that depend on the plate separation are decomposed into homogeneous and perturbed components. In this way, we solve the homogeneous components in a similar fashion to [20] and the perturbed components using a robust radial basis function meshless approach in order to characterise the effect of the variable plate separation from the homogeneous case.

Firstly, the plate separation can be represented with homogeneous and perturbed components as:

$$
b(\boldsymbol{x})=\bar{b}+\hat{b}(\boldsymbol{x})
$$

The perturbed component, $\hat{b}(\boldsymbol{x})$ represents the variation in $b(\boldsymbol{x})$ with respect to the homogeneous separation (the characteristic value defined at the origin) and the corresponding variation in permeability from the homogeneous base value in a porous medium. This gives the mobility of the fluid in zone $l$ as:

$$
\begin{aligned}
& M_{l}(\boldsymbol{x})=\frac{(\bar{b}+\hat{b}(\boldsymbol{x}))^{2}}{12 \mu_{l}}=\bar{M}_{l}(\boldsymbol{x})+\hat{M}_{l}(\boldsymbol{x}) \\
& \bar{M}_{l}=\frac{\bar{b}^{2}}{12 \mu_{l}}, \quad \hat{M}_{l}(\boldsymbol{x})=\frac{\hat{b}^{2}(\boldsymbol{x})+2 \bar{b} \hat{b}(\boldsymbol{x})}{12 \mu_{l}}
\end{aligned}
$$


In equation (4) the plate separation $b(\boldsymbol{x})$ can now be incorporated into a new modified mobility term:

$$
\begin{gathered}
m_{l}(\boldsymbol{x})=b(\boldsymbol{x}) M_{l}(\boldsymbol{x})=\frac{(\bar{b}+\hat{b}(x))^{3}}{12 \mu_{l}}=\bar{m}_{l}+\hat{m}_{l}(\boldsymbol{x}) \\
\bar{m}_{l}=\frac{\bar{b}^{3}}{12 \mu_{l}}, \quad \hat{m}_{l}(\boldsymbol{x})=\frac{\hat{b}^{3}(\boldsymbol{x})+3 \hat{b}^{2}(\boldsymbol{x})+3 \bar{b}^{2} \hat{b}(\boldsymbol{x})}{12 \mu_{l}}
\end{gathered}
$$

We now introduce non-dimensional variables utilising the characteristic length, time, velocity, pressure and mobility of the problem, given by:

$$
\begin{aligned}
(\boldsymbol{x}, r, b) & =r_{0}\left(\boldsymbol{x}^{\prime}, r^{\prime}, b^{\prime}\right), \quad t=\frac{r_{0}^{2}}{Q} t^{\prime}, \\
u_{l} & =\frac{Q}{r_{0}} u_{l}^{\prime}, \quad P_{l}=\frac{Q}{\bar{M}_{2}} P_{l}^{\prime}, \quad\left(M_{l}, m_{l}\right)=\bar{M}_{2}\left(M_{l}^{\prime}, m_{l}^{\prime}\right) \quad l=1,2
\end{aligned}
$$

In equalities (14) - (15), apostrophes identify non-dimensional variables with $t$ as time and $\bar{M}_{2}$ as the the homogeneous mobility of the displaced fluid (corresponding to the homogeneous thickness $\bar{b}$ ). From this point onwards, the apostrophe of all non-dimensional variables will be dropped, and all variables will be assumed to be in their non-dimensional form unless otherwise stated.

Consistent with the above representation of the mobility, in order to analyse the flow effects arising from the perturbed plate separation $\hat{b}(\boldsymbol{x})$ the total pressure can be represented as a sum of homogeneous and perturbed components:

$$
P_{l}(\boldsymbol{x})=\bar{p}_{l}(\boldsymbol{x})+\hat{p}_{l}(\boldsymbol{x})
$$

Expanding (4), noting that $\partial \bar{m}_{l} / \partial x_{i}=0$, the following equation is obtained:

$$
m_{l}(\boldsymbol{x}) \frac{\partial^{2} \bar{p}_{l}}{\partial x_{i}^{2}}+m_{l}(\boldsymbol{x}) \frac{\partial^{2} \hat{p}_{l}(\boldsymbol{x})}{\partial x_{i}^{2}}+\frac{\partial \hat{m}_{l}(\boldsymbol{x})}{\partial x_{i}} \frac{\partial \hat{p}_{l}(\boldsymbol{x})}{\partial x_{i}}=-\frac{\partial \hat{m}_{l}(\boldsymbol{x})}{\partial x_{i}} \frac{\partial \bar{p}_{l}(\boldsymbol{x})}{\partial x_{i}}
$$

The homogeneous pressure is constrained to satisfy Laplace's equation, in order to characterise the case of uniform plate separation and the effects of variable plate separation. i.e.:

$$
\frac{\partial^{2} \bar{p}_{l}(\boldsymbol{x})}{\partial x_{i}^{2}}=0
$$

To derive the correct matching and boundary conditions for the pressure field at the interface and the far field, the homogeneous and perturbation pressures can be substituted into (5) and (6). The homogeneous pressure is subject to the following matching and asymptotic conditions:

$$
\begin{aligned}
\bar{p}_{1}(\boldsymbol{\xi})-\bar{p}_{2}(\boldsymbol{\xi}) & =\frac{1}{C a_{g}}\left(\frac{2}{b(\boldsymbol{\xi})}+\frac{\pi}{4} k(\boldsymbol{\xi})\right) \quad \text { for } \quad \boldsymbol{\xi} \in S \\
\bar{M}_{1}(\boldsymbol{\xi}) \frac{\partial \bar{p}_{1}(\boldsymbol{\xi})}{\partial n} & =\bar{M}_{2}(\boldsymbol{\xi}) \frac{\partial \bar{p}_{2}(\boldsymbol{\xi})}{\partial n} \text { for } \boldsymbol{\xi} \in S \\
\bar{p}_{2}(\boldsymbol{x}) & \rightarrow-\frac{1}{2 \pi} \ln (r)
\end{aligned}
$$

Here, $C a_{g}$ is the global capillary number, appearing due to the non-dimensional scaling of the problem. The global capillary number in (19) describes the ratio of viscous driving forces to surface tension forces in terms of the homogeneous mobility $\bar{M}_{2}$ and plate separation $\bar{b}$ at the origin:

$$
C a_{g}=\frac{12 \mu_{2} Q}{\gamma r_{0}}\left(\frac{r_{0}}{\bar{b}}\right)^{2}=\frac{r_{0} Q}{\gamma \bar{M}_{2}}
$$

where $r_{0}$ is the initial unperturbed interface radius. By considering the global conditions given by (5), (6) and (8), equation (17) is then subject to the following matching conditions at the 
interface, $S$, and asymptotic conditions near the origin and in the far field:

$$
\begin{aligned}
\hat{p}_{1}(\boldsymbol{\xi})-\hat{p}_{2}(\boldsymbol{\xi}) & =0 \quad \text { for } \boldsymbol{\xi} \in S \\
M_{1}(\boldsymbol{\xi}) \frac{\partial \hat{p}_{1}(\boldsymbol{\xi})}{\partial n} & =M_{2}(\boldsymbol{\xi}) \frac{\partial \hat{p}_{2}(\boldsymbol{\xi})}{\partial n}+\left(\beta \hat{M}_{2}(\boldsymbol{\xi})-\hat{M}_{1}(\boldsymbol{\xi})\right) \frac{\partial \bar{p}_{1}(\boldsymbol{\xi})}{\partial n} \text { for } \boldsymbol{\xi} \in S \\
\frac{\partial \hat{p}_{1}(\boldsymbol{x})}{\partial r} & \rightarrow 0 \\
\begin{array}{c}
\boldsymbol{x} \rightarrow 0 \\
\hat{p}_{2}(\boldsymbol{x}) \\
\boldsymbol{x} \rightarrow \infty
\end{array} & \rightarrow 0
\end{aligned}
$$

where $\beta=\bar{M}_{1} / \bar{M}_{2}=\bar{\mu}_{2} / \bar{\mu}_{1}$ is the mobility ratio. The flux matching condition in equation (24) has a homogeneous pressure term on the RHS of the equation. We note that here, because the plate separation $b(\boldsymbol{\xi})$ is continuous at the interface, $\beta \hat{M}_{2}(\boldsymbol{\xi})=\hat{M}_{1}(\boldsymbol{\xi})$, meaning there is no contribution from the homogeneous pressure. We include the term for clarity so that the total flux matching condition in equation (5) is clearly met by the addition of equations (20) and (24).

Asymptotic conditions (21), (25) and (26) ensure that the total pressure and total velocity tend toward that arising solely from the injection source near the origin and in the far field. Since $b(\boldsymbol{x} \rightarrow 0)=\bar{b}$, the velocity field is defined solely by the homogeneous solution arising from $\bar{p}(\boldsymbol{x})$ near the origin.

The perturbed pressure flux matching condition (24) can be scaled by the plate variation $b(\boldsymbol{\xi})$ (i.e. $m_{l}(\boldsymbol{\xi})$ will appear instead of $M_{l}(\boldsymbol{\xi})$ ) in order to maintain consistency with the effective diffusion in equation (17). In this way, the homogeneous pressure evaluates the flow in a constant plate separation, and the perturbed pressure the effect due to changes in geometry of the flow path.

The obtained values of $\bar{p}_{l}$ and $\hat{p}_{l}$ can be used to reconstruct the normal velocity at an interface point, $\boldsymbol{\xi}$, in order to track the fluid:

$$
\begin{aligned}
& U_{n}(\boldsymbol{\xi})=\bar{u}_{n}(\boldsymbol{\xi})+\hat{u}_{n}(\boldsymbol{\xi}) \\
& U_{n}(\boldsymbol{\xi})=-M^{1}(\boldsymbol{\xi}) \frac{\partial \bar{p}_{1}(\boldsymbol{\xi})}{\partial n}-M^{1}(\boldsymbol{\xi}) \frac{\partial \hat{p}_{1}(\boldsymbol{\xi})}{\partial n}
\end{aligned}
$$

The evaluation of the perturbed and homogeneous pressure requires discretisation of the domain and interface as well as the use of a coupled BE - RBF-FC numerical method, discussed in the next section.

\section{Numerical methods}

Here, we describe the solution procedure to evaluate the perturbed and homogeneous pressure components and the subsequent reconstruction of the interface velocity with equation (28). The numerical methods have largely been presented in previous works by the authors, so where appropriate we refer the reader to these.

The homogeneous pressure is solved using a boundary element method presented by [20] and [21], in which the interface is discretised with boundary elements for the solution of an integral equation; see the interface in Figure 2. The boundary element method described by [20] explicitly tracks the interface, providing greater accuracy compared to front capturing methods, at the expense of increased meshing computational complexity. Using a convergent (Neumann) series technique, the computational cost scales quadratically with the number of boundary elements, significantly reducing running times compared with traditional $L U$ solvers of fully populated BEM matrices. As the surface interface grows, the number of boundary elements are adaptively increased to maintain a target element size.

In this work, we use an indirect formulation of the boundary element method akin to [21], which provides an identical homogeneous pressure and velocity as the direct method in [20], whilst allowing more efficient evaluation of domain pressures and velocities which are required for the perturbed pressure equation (17). For full details on the indirect boundary element method, the reader is referred to $[21]$. 


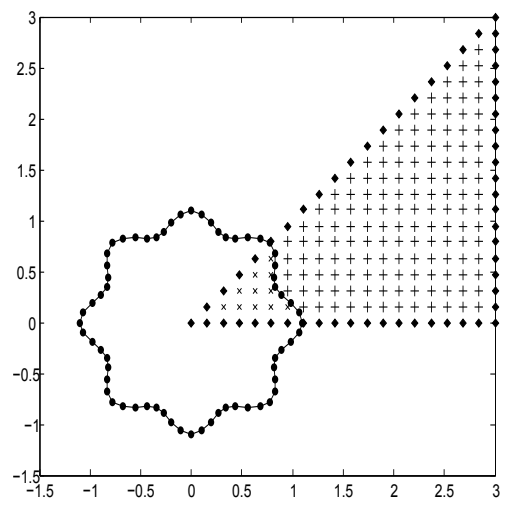

Figure 2: Discretised solution domain, with symbols referring to: circles - interface nodes (connecting boundary elements), diamonds - boundary nodes, crosses - internal zone nodes, plus signs - external zone nodes.

The perturbed pressure equation (17) is solved via a strong form radial basis - function finite collocation (RBF-FC) method which is also presented in [21]. Here, we require both internal and boundary nodes as seen in Figure 2 [22], which are adaptively added and removed from the domain based on the interface location using a quadtree data structure. To decrease the solution cost for this method we use an 8 finger symmetric interface throughout the numerical simulations; this enables the RBF-FC method to make use of symmetry in the domain and solve only $1 / 8^{\text {th }}$ of the entire domain.

We note that any form of symmetric could be used here to reduce computation time, but the conclusions drawn will be exactly the same. A change in wavelength and wavenumber of initial perturbation will alter the magnitude and time-scale at which fingering events occur, but the mechanisms for fingering (and stability) will remain unchanged. We choose an 8 finger symmetry, since it is efficient to split a quadtree dataset into eighths, as opposed to a 6 finger symmetry which would require displacement of nodes in the quadtree dataset so that they fall onto the line of symmetry.

In the work of [21], the RBF-FC method is formulated with adaptive quadtree datasets on single zone transport problems, which we extend in this work to multi-zone problems for the solution of the steady-state perturbed pressure in equation (17). By displacing nodes to coincide directly with the interface, two stencils can be formed at each interface node, so that multi-zone flux and solution matching conditions can be enforced. This global collocation of matching conditions is performed in the same manner as the multi-zone formulation in [23], where they use a finite difference (FD) approach to enforce the underlying PDE.

Here, we use a finite collocation approach to enforce the governing PDE, in which the PDE collocation only appears in the local systems and globally the solution variable is reconstructed. The RBF-FC method has been shown to have several benefits over the RBF-FD method, most importantly the implicit upwinding effect for strongly convective fields using centrally defined stencils [22]. Incorporating an adaptive dataset that evolves with the interface also significantly reduce computational time whilst maintaining solution accuracy [21].

The quadtree dataset is initialised using a distance based scheme to cluster cells around the boundary element interface, in which a cell will split if the following equality is met: $D_{c} B_{t}>R_{c}$. Here, $D_{c}$ refers to the diagonal length of the cell, $R_{c}$ is the distance from the cell centre to the closest point on the interface and $B_{t}$ is the band thickness. The band thickness refers to the 'bands' of constant nodal discretisation that are formed around the interface. Increasing $B_{t}$ creates more nodes at each particular level, creating wider bands. After initialisation, the cells closest to the interface are deformed such that their vertices lie directly on the interface and the centres lie at the geometric centre of the cell. Vertices move in a purely orthogonal direction to maintain spatial consistency.

After the quadtree has been generated, the nodal points for the RBF-FC local systems can be created based on the vertices and cell centres of the leaf cells. A simple tree search can be performed, with nodal locations picked out as the tree is recursively traversed, and grouped into local stencil configurations. At interior and boundary stencils, we use the 1-1 stencil configuration 


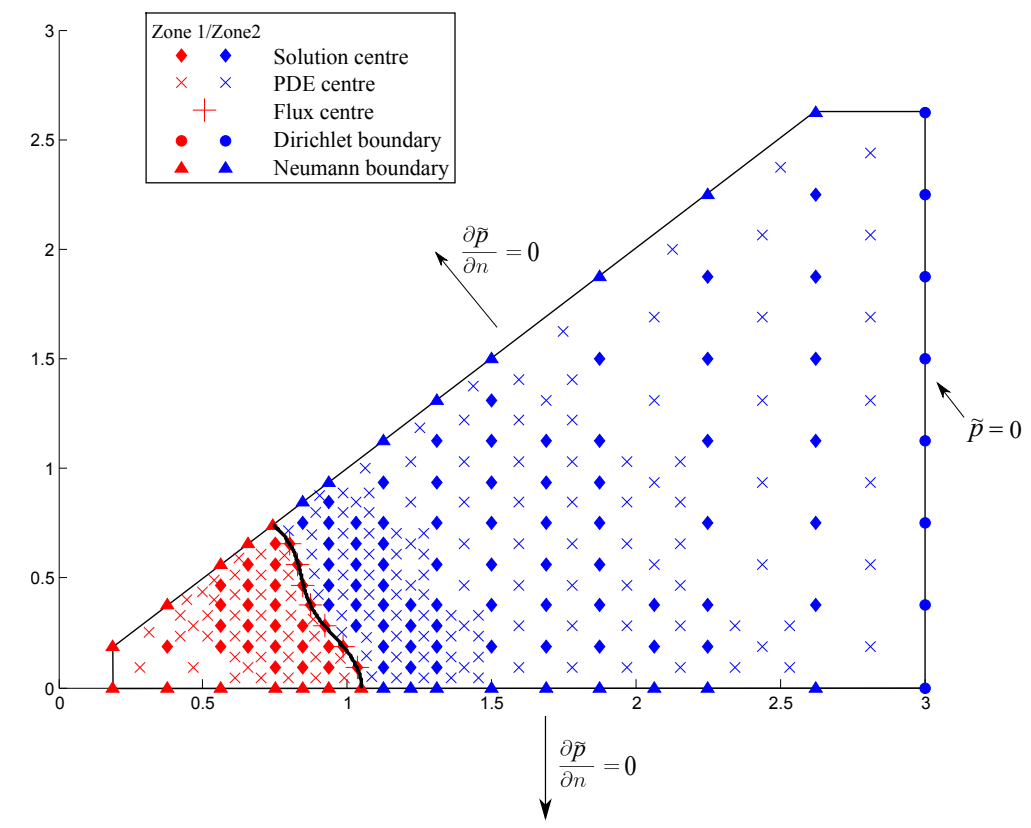

Figure 3: RBF-FC solution domain showing different operator types. The curved solid black line indicates the fluid-fluid interface.

presented in [21]. For multi-zone stencils, we form two stencils at each interface node, which follow the same 1-1 configuration, but extend purely into the zone of influence. This means the number of nodal points can vary, depending on the topology of the interface, but generally varies between 11 and 17. Extension of the stencils as opposed to truncation is preffered to maintain solution accuracy, see [22].

After formation of the local stencils and global collocation of the solution variable (the perturbed pressure in this case), the sparse global matrix system can be solved with any direct LU solver. The perturbed pressure solution can then be fed back into the data vectors in the local systems, which can then be used to reconstruct the perturbed pressure gradients to evaluate the velocity at the interface.

The full numerical algorithm for solution of the homogeneous and perturbed pressures and subsequent evaluation of the normal interface velocity is summarised below:

1. Initialise the interface with an 8 finger perturbation and discretise into B-Spline boundary elements.

2. Initialise the quadtree dataset, deforming it around the initial interface position.

3. Solve the double layer potential density around the interface and find the homogeneous pressure along the interface..

4. Reconstruct the normal homogeneous velocity around the interface by taking the spatial derivatives of the double layer potential and reconstruct the source term for equation (17) at every internal node.

5. Solve equation (17) using the RBF-FC method and reconstruct the normal perturbation velocity around the interface.

6. Find the total normal velocity at the interface using equation (27), and advance the boundary element nodes using a forward Euler time step scheme: $\Delta x_{i}=n_{i} U_{n} \Delta t$.

7. Re-form the boundary elements around the new interface position. Adapt the quadtree dataset to the new interface position. Increase time by $\Delta t$.

8. Repeat steps 3 to 7 until the simulation end time has been reached.

\section{Mass conservation verification}

In this section, we demonstrate the full numerical scheme on the case of the displacement of an initially circular interface, with zero perturbation. Perturbations will only grow after a long period 
of time due to numerical error, meaning that the interface should propagate as a growing circle with an increasing volume equal to that injected at the origin. By comparing the numerical volume of the growing plume and the analytical volume injected at the origin, we can verify that the coupled scheme is mass conservative (since the density is constant) and that the governing equation of the flow (4) is being satisfied. The total volume of injected fluid is given in non-dimensional form as:

$$
V_{a}=\bar{b} t
$$

To ensure that mass has been conserved in the numerical method, the total volume of injected fluid can be calculated through numerical integration of the evolving interface (using the average interface position $\bar{r}$ ) and compared to the analytical value given by equation (29). Although there are no fingering effects present due to the constant curvature, the problem still provides validation of the coupling of the RBF method and the BEM. The BEM has been verified for homogenous mobility cases in previous work by [20], meaning the mass conservation tests presented here will verify that the RBF-FC method is contributing a correct velocity to displace the interface. The RBF method still solves the full multi-zone steady-state pressure problem, but with the interface given by a simple circle instead of the convoluted interface usually found in viscous fingering problems.

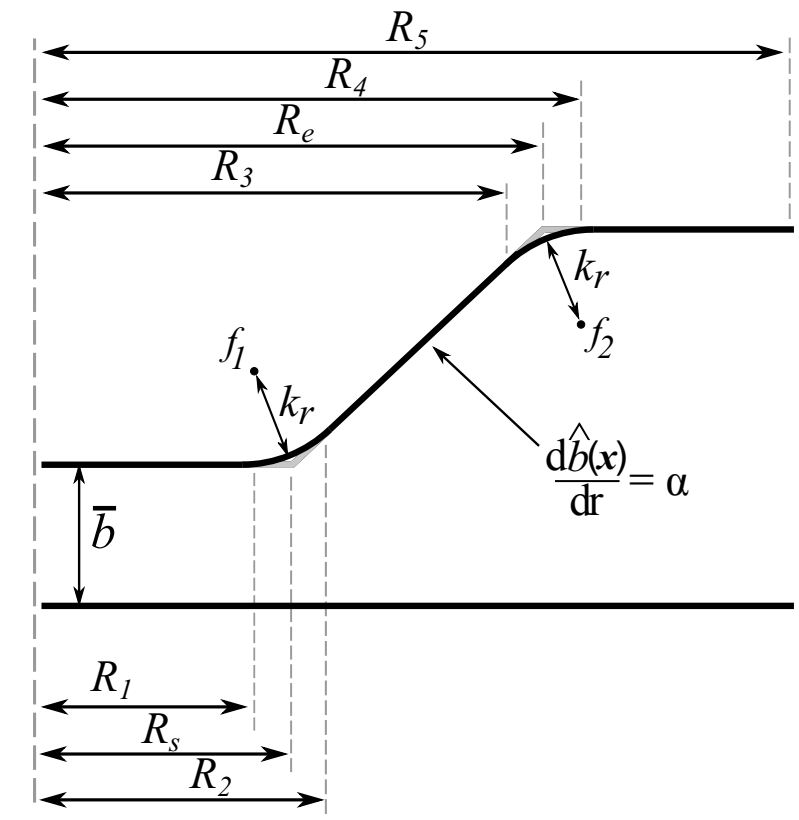

Figure 4: Gradually diverging Hele-Shaw cell diagram (gradient exaggerated for display purposes). The dashed line at the far left of the domain shows the line of symmetry at the origin.

For a variable permeability test case we study the gradually varying cell geometry in Figure 4. The choice of variation is chosen so as to mimic the uniformly converging/diverging geometries used by previous authors [8]. Although the cell is not uniformly converging/diverging along its whole length, the constant slope section is made long enough so that the interface displaces within it for the entire duration of the simulation, effectively mimicking uniform variation. Using the uniformly varying cell, we can compare the results across a range of capillary numbers with that given by previous authors.

The cell is axisymmetric around the origin, with values of $\hat{b}(\boldsymbol{x})$ and $\partial \hat{b}(\boldsymbol{x}) / \partial x_{i}$ at the different radial locations $r=|\boldsymbol{x}|$ in Figure 4 as: 


$$
\begin{array}{rlrl}
\hat{b}(\boldsymbol{x}) & =0, \quad \frac{\partial \hat{b}(\boldsymbol{x})}{\partial x_{i}}=0 \quad r<R_{1}, r>R_{4} & & \\
\hat{b}_{l}(\boldsymbol{x}) & = \pm\left(R_{w}^{2}-\left(r-f_{l}(1)\right)^{2}\right)^{\frac{1}{2}}+f_{l}(2)-\bar{b}, & \frac{\partial \hat{b}(\boldsymbol{x})}{\partial x_{i}}=\mp\left(\frac{\left(\frac{x_{i}}{r}\right)\left(r-f_{l}(1)\right)}{\left(R_{w}^{2}-\left(r-f_{l}(1)\right)^{2}\right)^{\frac{1}{2}}}\right) \\
R_{1}<r<R_{2}, R_{3}<r<R_{4} & \\
\hat{b}(\boldsymbol{x}) & =\alpha\left(r-R_{s}\right), \quad \frac{\partial \hat{b}(\boldsymbol{x})}{\partial x_{i}}=\alpha\left(\frac{x_{i}}{r}\right) & R_{2}<r<R_{3}
\end{array}
$$

In equation (31), subscript $l$ refers to fillet 1 or 2 in Figure $4 . f_{l}$ is the centrepoint of fillet $l$ with coordinates $\left(f_{l}(1), f_{l}(2)\right)$, with radius of curvature $k_{r}$. The $+/-\operatorname{sign}$ in $\hat{b}_{l}(\boldsymbol{x})$ refers to a converging/diverging fillet $\left(2^{n d} / 1^{\text {st }}\right.$ fillet in Figure 4$) . R_{w}$ is the length of the fillet, i.e., $R_{w}=$ $R_{s}-R_{1}$. The fillet is defined by inputs of $R_{s}, R_{e}, R_{w}$ and simple trigonometric identifies. In the simulations in this section $\bar{b}=0.01$, the gradient of the slope $|\alpha|=0.001, R_{s}=0.6, R_{e}=5$ and $R_{w}=0.1$. These parameters ensure that plate separation varies gradually through the cell and that the circular interface, with an unperturbed radius of $r_{0}=5 \mathrm{~cm}$, exists entirely in the constant gradient section from $t=0$ to the end of the simulation.

Given the symmetry of the problem, we use a triangular solution domain for the RBF-FC method, similar to that shown in Figure 3, with an outer boundary at $x_{1}=32$. For the validation cases we vary the maximum quadtree cell level from 8 - 10 whilst maintaining a minimum quadtree cell level of 3 . The band thickness values are varied for each cell level, with the maximum cell level having $B_{t}=3$. This value is increased in increments of 0.5 up to the minimum cell level. We maintain a constant non-dimensional shape parameter value of $c^{*}=70$ for all simulations. The time step size is varied to showcase the temporal convergence properties. In the boundary element method the full interface is solved, around which a target element size is maintained throughout the simulations at $\Delta x \approx 0.06$, ensuring a mesh-independent boundary element solution.

\begin{tabular}{|l|l|}
\hline Property & Value (SI Units) \\
\hline$\mu_{\text {Brine }}$ & $1 \times 10^{-3}$ Pa.s \\
\hline$\mu_{C O_{2}(s c)}$ & $1 \times 10^{-4} \mathrm{Pa.s}$ \\
\hline$\mu_{\text {MineralOil }}$ & $2.5 \times 10^{-2} \mathrm{Pa.s}$ \\
\hline$\mu_{\text {Air }}$ & $2 \times 10^{-5} \mathrm{~Pa} . \mathrm{s}$ \\
\hline$\beta C \mathrm{O}_{2}(\mathrm{sc}) /$ brine & 10 \\
\hline$\beta$ Air/Mineral Oil & 1250 \\
\hline$\gamma\left(\mathrm{CO}_{2}\right.$-Brine $) \& \gamma$ (Air-Mineral Oil) $)$ & $0.03 \mathrm{~kg} / \mathrm{s}^{2}$ \\
\hline
\end{tabular}

Table 1: Fluid properties used in the numerical experiments

The fluid properties used for the numerical experiments in both this section and the results section are summarised in table 1 . In this validation study, we use $\mathrm{CO}_{2}$ and brine as the working fluids, with a global capillary number $C a_{g}=1000$.

The results showing the relative error between the numerical and analytical volumes of fluid in the Hele-Shaw cells for various $\alpha$ at time $t=10$ are displayed in table 2. It can be seen that the relative errors for all time step and mesh discretisations shown are relatively low, with the largest error being $1.42 \times 10^{-2}$ for the $\alpha=-0.0025$ case. In this case, the sharply converging cell causes significant acceleration of the interface which creates a large amount of error in the time stepping scheme. The datasets with their maximum quadtree level (MQL) equal to 8 show the largest error generally, due to the very coarse discretisation around the interface. With MQL $=8$, the RBF dataset is roughly $2 \mathrm{x}$ coarser then the boundary element mesh, meaning that several elements can exist between RBF nodes. This is undesirable as the interface could curve significantly between data points, meaning the interface matching conditions are not adequately represented in the RBF dataset. As the nodal points are 'snapped' to the nearest position on the boundary element mesh, the resulting RBF dataset is not as uniform if the nodal discretisation is very much larger than the boundary element discretisation. 
When the MQL is increased from 8 through to 10, the relative error drops significantly, especially at the smaller time step sizes. At the lowest time step size of $\Delta t=0.005$, the error is 9 to 16 times lower than at $\mathrm{MQL}=8$, representing close to $2^{\text {nd }}$ order spatial convergence. The increased solution quality is due to the MQL $=10$ dataset being twice as refined as the boundary element mesh around the interface. This means that in between the RBF nodes, the interface is much closer to a linear approximation, making the strong form RBF collocation of matching conditions more consistent, leaving less space for the flux and solution matching conditions to vary between points. At $\mathrm{MQL}=10$, the time stepping scheme exhibits 1st order convergence as expected due to the forward Euler implementation.

In some instances, the error can be seen to plateau due to the spatial or temporal accuracy of the scheme being reached. This is apparent in some diverging cases with the same time step size, when increasing MQL from 9 to 10 has not caused a reduction in error, since it is limited by the temporal accuracy. A significant reduction in time step size would be needed to see an increase in accuracy for these cases. Similarly, in the converging case with $\alpha=-0.025$, the spatial accuracy has been reached when using relatively large time step sizes, meaning no further reduction increases the accuracy. To increase the accuracy in these cases, the spatial resolution has to be increased significantly.

\begin{tabular}{|c|c|c|c|c|}
\hline \multirow{3}{*}{$\begin{array}{l}\text { Hele-Shaw } \\
\text { cell case }\end{array}$} & \multirow{3}{*}{$\begin{array}{c}\text { time step } \\
\text { size, } \Delta \mathrm{t}\end{array}$} & $\mathrm{MQL}=8$ & $\mathrm{MQL}=9$ & $\mathrm{MQL}=10$ \\
\hline & & $\Delta \mathrm{x}=0.125$ & $\Delta \mathrm{x}=0.0625$ & $\Delta \mathrm{x}=0.03125$ \\
\hline & & Relative error & Relative error & Relative error \\
\hline \multirow{4}{*}{$\begin{array}{l}\text { Converging } \\
\alpha=-0.001\end{array}$} & 040 & $3.97 \times 10^{-3}$ & $2.14 \times 10^{-3}$ & $1.29 \times 10^{-3}$ \\
\hline & 20 & $3.35 \times 10^{-3}$ & $1.51 \times 10^{-3}$ & $6.60 \times 10^{-4}$ \\
\hline & 0.010 & $3.05 \times 10^{-3}$ & $1.18 \times 10^{-3}$ & $3.45 \times 10^{-4}$ \\
\hline & 0 . & $2.90 \times 10^{-3}$ & $1.04 \times 10^{-3}$ & $1.87 \times 10^{-4}$ \\
\hline \multirow{4}{*}{$\begin{array}{l}\text { Converging } \\
\alpha=-0.025\end{array}$} & & $1.22 \times 10^{-2}$ & $5.29 \times 10^{-3}$ & $5.19 \times 10^{-3}$ \\
\hline & 0.020 & $1.28 \times 10^{-2}$ & $5.87 \times 10^{-3}$ & $4.25 \times 10^{-3}$ \\
\hline & & $1.35 \times 10^{-2}$ & $4.48 \times 10^{-3}$ & $4.50 \times 10^{-3}$ \\
\hline & & $1.42 \times 10^{-2}$ & $5.99 \times 10^{-3}$ & $5.54 \times 10^{-3}$ \\
\hline \multirow{4}{*}{$\begin{array}{l}\text { Diverging } \\
\alpha=0.001\end{array}$} & 0. & $3.02 \times 10^{-3}$ & $9.46 \times 10^{-4}$ & $1.56 \times 10^{-3}$ \\
\hline & 0 . & $2.22 \times 10^{-3}$ & $1.77 \times 10^{-4}$ & $7.57 \times 10^{-4}$ \\
\hline & .010 & $1.83 \times 10^{-3}$ & $2.02 \times 10^{-4}$ & $3.67 \times 10^{-4}$ \\
\hline & 05 & $1.64 \times 10^{-3}$ & $4.02 \times 10^{-4}$ & $1.78 \times 10^{-4}$ \\
\hline \multirow{4}{*}{$\begin{array}{l}\text { Diverging } \\
\alpha=0.025\end{array}$} & 0 & $9.39 \times 10^{-3}$ & $6.10 \times 10^{-4}$ & $1.59 \times 10^{-3}$ \\
\hline & & $8.39 \times 10^{-3}$ & $7.38 \times 10^{-4}$ & $7.53 \times 10^{-4}$ \\
\hline & & $.02 \times 10^{-3}$ & $1.18 \times 10^{-3}$ & $3.60 \times 10^{-4}$ \\
\hline & & $8.30 \times 10^{-3}$ & $1.35 \times 10^{-3}$ & $1.46 \times 10^{-4}$ \\
\hline \multirow{4}{*}{$\begin{array}{l}\text { Uniform } \\
\alpha=0.000\end{array}$} & & $1.43 \times 10^{-3}$ & $1.43 \times 10^{-3}$ & $1.43 \times 10^{-3}$ \\
\hline & & $7.16 \times 10^{-4}$ & $7.16 \times 10^{-4}$ & $7.16 \times 10^{-4}$ \\
\hline & 0.010 & $3.58 \times 10^{-4}$ & $3.58 \times 10^{-4}$ & $3.58 \times 10^{-4}$ \\
\hline & 0.005 & $1.79 \times 10^{-4}$ & $1.79 \times 10^{-4}$ & $1.79 \times 10^{-4}$ \\
\hline
\end{tabular}

Table 2: Relative errors between the numerical and analytical volumes of fluid in the cell at $t=10$ with different mesh and time step discretisations. MQL = maximum quadtree cell level.

The time evolution of the relative errors between the numerical and analytical volume of fluid for the $\alpha=-0.001$ cases can be seen in Figure 5. For a fixed low time step of $\Delta t=0.005$ in Figure $5 \mathrm{a}$, the error for the different nodal discretisations becomes much more consistent as $\Delta x$ is lowered. The coarse discretisation of $\Delta x=0.125(\mathrm{MQL}=8)$, shows fairly erratic behaviour due to the interface representation. However the error evolution becomes much smoother as $\Delta x \rightarrow 0.03125$. As time progresses the error drops since the interfacial velocity becomes lower and the fixed Euler time stepping scheme becomes more accurate.

The first order temporal convergence can be seen in Figure 5b, where the time step size is varied for the finest spatial discretisation $(\Delta x=0.03125)$. Due to the smoothness of the error evolution and the generally low relative error, we choose the finest dataset $(\Delta x=0.03125$, MQL $=10)$ for simulations in the following results sections. For practicality, we use $\Delta t=0.02$, to provide a good balance between solution accuracy and simulation running times. For these parameters, the largest 


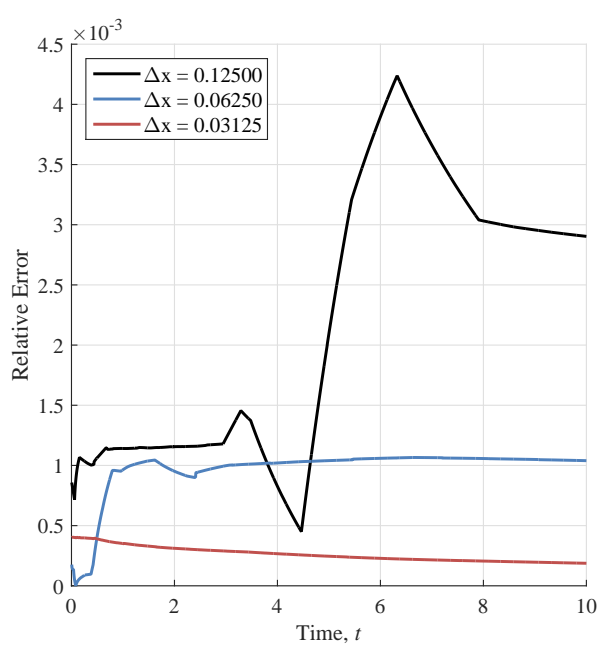

(a)

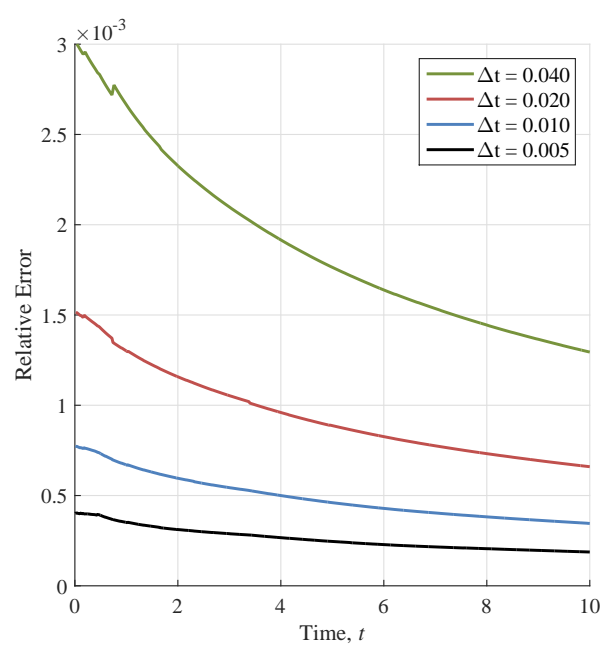

(b)

Figure 5: Time evolution plots of the relative error between analytical and numerical volume of fluid in the converging Hele-Shaw cell $\alpha=-0.001$. (a) Different nodal discretisations, $\Delta t=0.005$. (b) Different time step discretisations, $\Delta x=0.03125$.

error in the above verification examples is only $4.25 \times 10^{-3}$, showing the proposed accuracy of the parameters chosen.

\section{Numerical results and discussion}

In this section we present results from several numerical experiments exploring the variable plate separation problem. In order to compare with previous work, initially we present studies based on air injection into mineral oil, corresponding to the cases of negligible viscosity of the injected fluid considered by [9], [11] and [15]. In the present cases, the low viscosity of the injected fluid is included in the model, resulting in a (high) finite mobility ratio between the air and mineral oil. As well as the high mobility ratio cases, a lower mobility ratio regime is considered in order to analyse the injection of supercritical $\mathrm{CO}_{2}$ into brine. The fluid properties used for the numerical experiments are summarised in the previous section in table 1.

Throughout the results, we present expressions and contour plots showing the variation in plate separation $\hat{b}(\boldsymbol{x})$ as opposed to the variation in cell permeability $\hat{k}(\boldsymbol{x})=\left(\hat{b}^{2}(\boldsymbol{x})+\bar{b} \hat{b}(\boldsymbol{x})\right) / 12$ (the homogeneous permeability is $\left.\bar{k}=\bar{b}^{2} / 12\right)$. This is purely because the plate separation itself is varied in order to change the permeability, and it is easier to view the gradually varying plate separation variation. All plate separation values can be scaled using the above expression to form the appropriate variation in permeability if needed.

Throughout the numerical simulations, we use an 8 finger symmetric pattern as the initial interface condition, given in Figure 1a as:

$$
r=1+\epsilon_{0} \cos (8 \theta)
$$

where $\epsilon_{0}$ and $\theta$ are the perturbation amplitude and the azimuthal angle around the interface respectively.

\subsection{Gradually converging/diverging Hele-Shaw cells}

This section explores the immiscible displacement in a gradually varying cell geometry with a small constant gradient $\alpha$ in the radial direction, as shown in Figure 4 in the previous section. In the simulations in this section $\bar{b}=0.01, R_{s}=0.6, R_{e}=5$ and $R_{w}=0.1$. The initial perturbation amplitude $\epsilon_{0}=0.05$. These parameters ensure that plate separation varies gradually through the cell and that the interface, with an unperturbed radius of $r_{0}=5 \mathrm{~cm}$, exists entirely in the constant gradient section from $t=0$ to the end of the simulation. 
Given the symmetry of the problem, we use a triangular solution domain for the RBF-FC method, similar to that shown in Figure 3, with an outer boundary at $x_{1}=32$. The minimum quadtree cell level is 3 with a maximum level of $10, B_{t}$ varies uniformly from 3 at cell level 10 to 6.5 at cell level 3 and $c^{*}=70$. In the boundary element method the full interface is solved, around which a target element size is maintained throughout the simulations at $\Delta x \approx 0.06$ and time step size of $\Delta t=0.02$. This ensures that the RBF-FC nodal refinement is around twice that of the BEM around the interface, and that both pressure solutions are mesh and time step independent.

We break the analysis into subsections to aid discussion, starting with the general system behavior below.

\subsubsection{General system behaviour}

In Figure 6 we present results for different capillary number and mobility ratio flows in uniform, converging and diverging cell geometries. $\beta=1250$ corresponds to air injection into mineral oil, and $\beta=10$ corresponds to supercritical $\mathrm{CO}_{2}$ injection into brine. In the converging cases in Figures $6 \mathrm{a}$ and $6 \mathrm{~d}$, the interfaces have been stabilised in the low capillary number regimes, where the bases of the fingers have been pushed outwards, resulting in more circular interfaces. The lower mobility ratio in Figure 6d has stabilised the interface even further compared to Figure 6a. This is due to the higher finger base velocities present in low mobility ratio cases, as the inner fluid has a non-negligible viscosity. This, coupled with the converging cell geometry, produces a very stable interface that shows almost no signs of the usual viscous fingering regime. The stabilising effect of the converging geometry is also present in Figures $6 \mathrm{~b}$ and $6 \mathrm{e}$; however, with an increasing capillary number the stabilisation becomes less prominent.

The stabilisation in the low capillary number regime in the converging cases is due to the dominating effect of the transverse curvature in the capillary pressure jump condition. Here, as $C a_{g}$ is taken lower, the $2 / b(\boldsymbol{x})$ transverse curvature term has more effect. Parts of the interface which are further back in the plane of the cell are at a larger plate separation than those further forward, and hence the $2 / b(\boldsymbol{x})$ term is smaller. The increase in transverse curvature is effectively creating a positive gradient in capillary pressure through the cell. This capillary pressure gradient overcomes the viscous pressure gradient to control the flow and smooth the interface, working against the viscous fingering mechanism.

At the higher capillary numbers, the effects of the capillary pressure gradient are smaller, and the interfaces in the converging cases can be seen to displace beyond the uniform cases in Figures $6 \mathrm{c}$ and $6 \mathrm{f}$. The magnitude of the normal pressure gradient $|\partial P(\boldsymbol{x}) / \partial n|$ increases in the converging section to ensure mass conservation, directly competing with the stabilisation from the capillary pressure and the reduction in fluid mobility through the converging section. The converging cell therefore induces the following effects that control the stability of the displacing interface:

1. Stabilisation through an increased transverse curvature (positive capillary pressure gradient).

2. Deceleration due to a reduced fluid mobility.

3. Acceleration due to an increased (viscous) pressure gradient.

These effects can be thought of as aperture effects, as they result directly from the spatial change in cell aperture (plate separation). In Figure 6 we can see that the combined effects of the converging geometry appears to transition from stabilising at a low capillary number (aperture effect 1 and 2 dominate), to destabilising at a high capillary number (aperture effect 3 dominates). These effects are relative in relation to the uniform cell cases, i.e., at lower capillary numbers the interface is stabilised in comparison to the uniform cell, but it may still be 'unstable' in an absolute sense and exhibit viscous fingering instabilities. We refer to this as 'relative stability', i.e. the converging cell interface in figure $6 \mathrm{a}$ is relatively more stable than the uniform cell interface.

In contrast to the converging cases, the aperture effects listed above are exactly opposite for the diverging cases; the diverging cells in Figures $6 \mathrm{a}$ and $6 \mathrm{~d}$ create a more convoluted interface in the low capillary number regime, but smooth the interface in the high capillary numbers regime in Figures $6 \mathrm{c}$ and $6 \mathrm{f}$. The transition in relative stability in both converging and diverging cases corresponds to a change in the flow regime from capillary dominated to viscous dominated.

The aperture effects of the converging/diverging Hele-Shaw cells are further highlighted by the different components of the solution in Figure 7. The difference between the solid lines and the dashed lines shows the effect of the stabilising $2 / b(\boldsymbol{x})$ term (i.e capillary pressure). The solid 


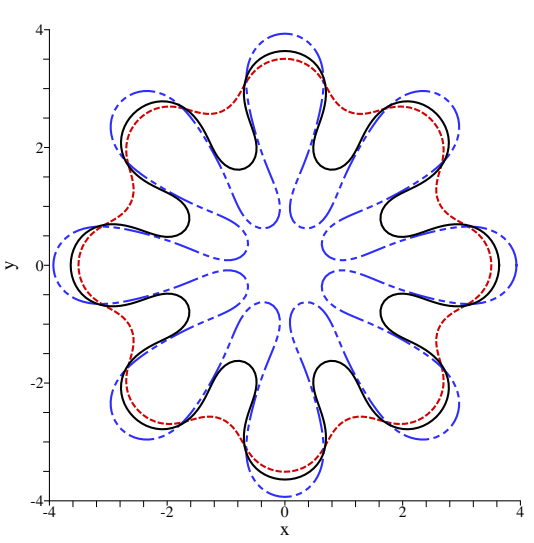

(a) $\beta=1250, C a_{g}=500$

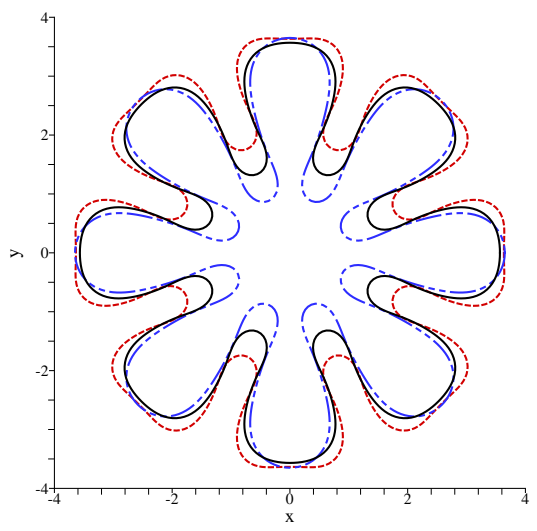

(b) $\beta=1250, C a_{g}=1000$

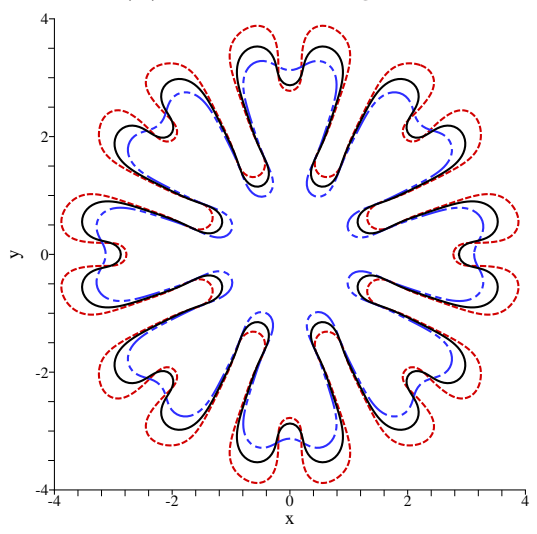

(c) $\beta=1250, C a_{g}=2500$

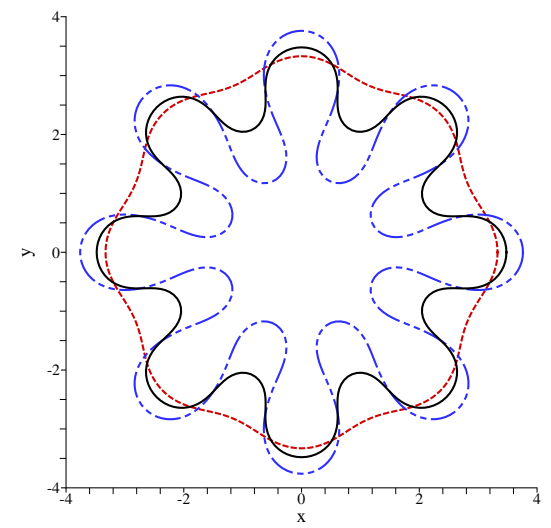

(d) $\beta=10, C a_{g}=500$

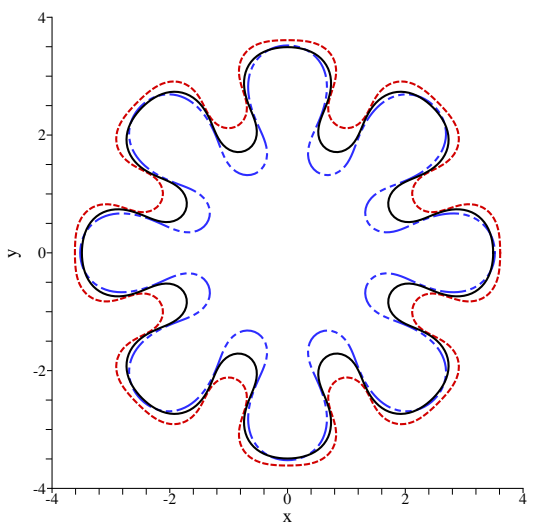

(e) $\beta=10, C a_{g}=1000$

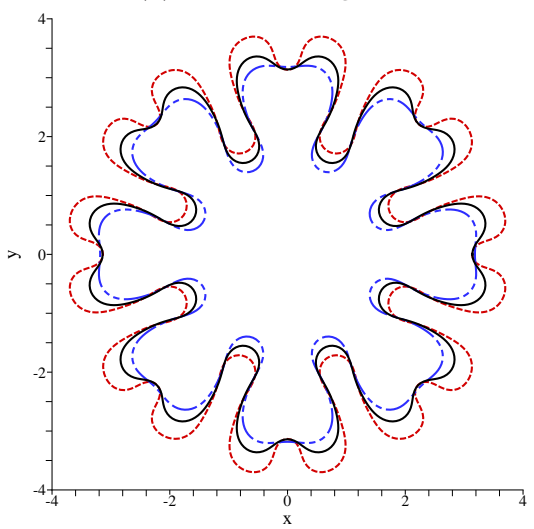

(f) $\beta=10, C a_{g}=2500$

Figure 6: Interface plots at $t=24$ for different cell geometries. $|\alpha|=0.001$. - Uniform cell, --- Converging cell, - - - Diverging Cell

lines have the transverse curvature term included in the capillary pressure jump, and as such the interface is smoothed from the dashed lines in the converging case $7 \mathrm{~b}$, and destabilised in the diverging case $7 \mathrm{a}$.

The effect of the change in (viscous) pressure gradient can also be seen in Figure 7 by considering the solution with and without the perturbed pressure component. Without the perturbed pressure component, the change in pressure gradient due to the converging/diverging geometry (aperture effect 3 ) is not included. This means the interface velocity consists of the uniform cell pressure gradient with a variable mobility and transverse curvature (solely aperture effects 1 and 2). Figure $7 \mathrm{~b}$ shows that the black interfaces have all been displaced beyond the grey interfaces which do not 


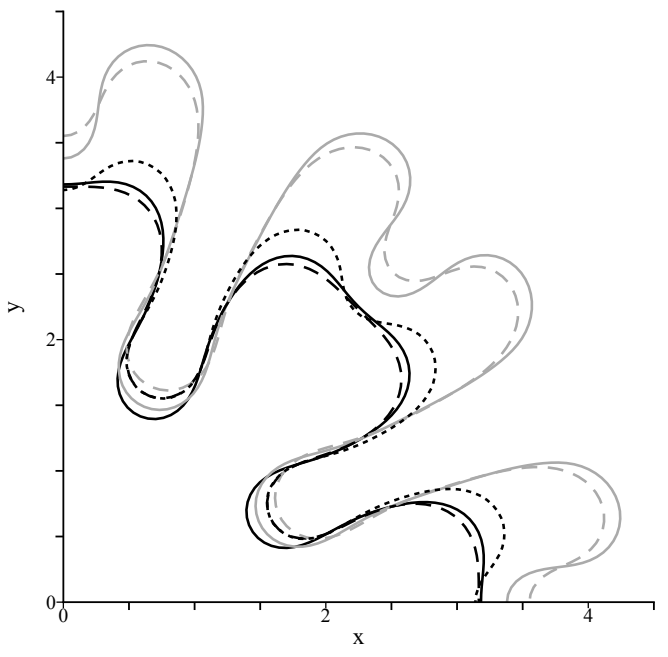

(a) $\alpha=0.001$

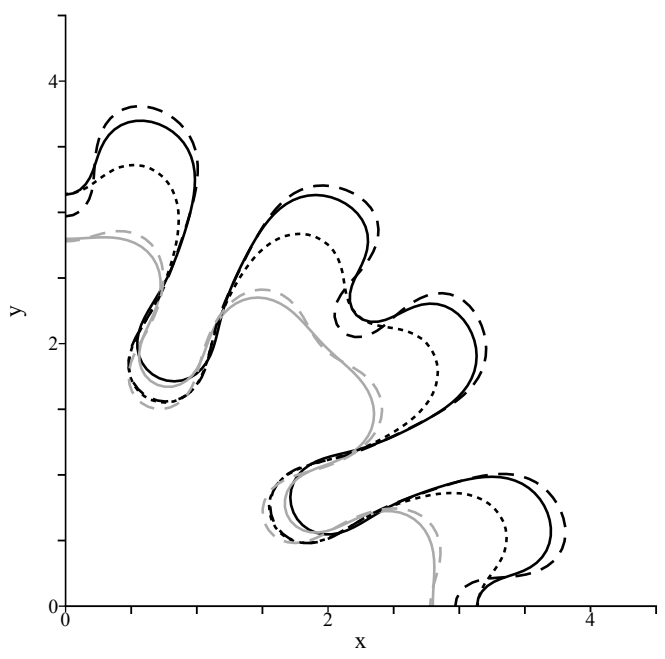

(b) $\alpha=-0.001$

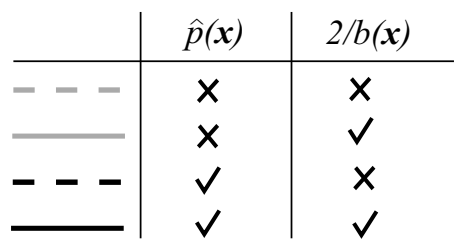

Legend

Figure 7: (a) and (b) - Solution component plots at $t=24$. Ticks in the legend indicate which interfacial displacements in (a) and (b) include the curvature term $2 / b(\boldsymbol{x})$ and the perturbed pressure $\hat{p}(\boldsymbol{x})$. The dotted lines represent the uniform cell case. $\beta=10, C a_{g}=2500$.

include the perturbed pressure. The converging geometry creates a decreasing perturbed pressure, whose gradient accelerates the interface. Without the perturbed pressure, the grey interfaces are not displaced even as far as the uniform cases, showing the dramatic effect that the increased pressure gradient has on the interfacial evolution at this high capillary number. The inclusion of the perturbed pressure has only significantly affected the finger fronts, with the base position remaining relatively unchanged, being affected more significantly by the transverse curvature. The accelerating flow from the perturbed pressure in the converging case has made the finger bifurcate earlier. In the diverging case, the perturbed pressure gradient slows the interface, helping to stabilise it and hinder bifurcation; clearly visible in Figure 7a.

The increased flattening and bifurcation present in the converging cases here is in contrast to the findings of [9] in a rectilinear converging cell, whereby the finger shape was found to sharpen. This contrast is due to the rectilinear geometry, where one long prominent finger is produced with a uniform width throughout its length, roughly equal to half the cell width. In [9], the plate separation varies solely along the length of the cell. When the long finger is formed in a converging cell, the front is sharpened, since the tip is accelerated more strongly in comparison to the rest of the finger. However, in the case of a constant radial variation in the plate separation, the sides of the fingers are accelerated in a similar manner to the finger tips, and the front becomes flatter promoting bifurcation. The contrast in results is due to the variation in the plate separation with respect to the flow path of the fingers. In radial displacement, the finger tips and sides displace into pathways of similar convergence, whereas in rectilinear flow, the tip evolves in a much more converging pathway than the sides, sharpening the finger. These differences are discussed in more detail in $§ 5.3$.

\subsubsection{The transition in relative stability}

We analyse the transition in relative stability for the converging/diverging cases between low and high capillary numbers by considering the growth rate of the finger front perturbation, which can highlight the relative stability between cases. The dimensionless non-linear growth rate of a 
perturbation with radial position $r$ is given by:

$$
\sigma_{t}=\left(\frac{U_{n}(r, t)-U_{n 0}(r, t)}{\epsilon_{0} U_{n 0}(r, t)}\right)\left(\frac{\beta+1}{\beta}\right)
$$

where, $U_{n}(r, t)$ is the normal velocity at the perturbed interface and $U_{n 0}(r, t)$ is the normal velocity at the unperturbed interface (in a uniform cell). The perturbation amplitude $\epsilon_{0}$ and the mobility ratio $\beta$ are used as scalings to be consistent with the linear stability equation of [8] presented later. The velocity of the unperturbed interface with time is given purely by the source injection velocity at the corresponding radius, $r=\sqrt{\frac{t+\pi}{\pi}}$. The non-linear growth rate (34) subtracts the linear velocity of the base growth giving the non-linear growth of the perturbation with time. The non-linear growth rate for the finger fronts (using the rightmost finger in each plot in Figure 6 along $y=0$ ) has been plotted with time for the $C a_{g}=500,1000$ and 2500 cases in Figure 8.

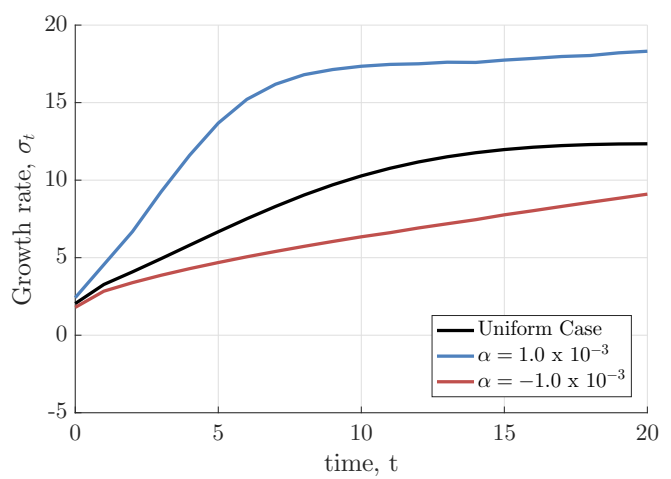

(a) $C a_{g}=500$

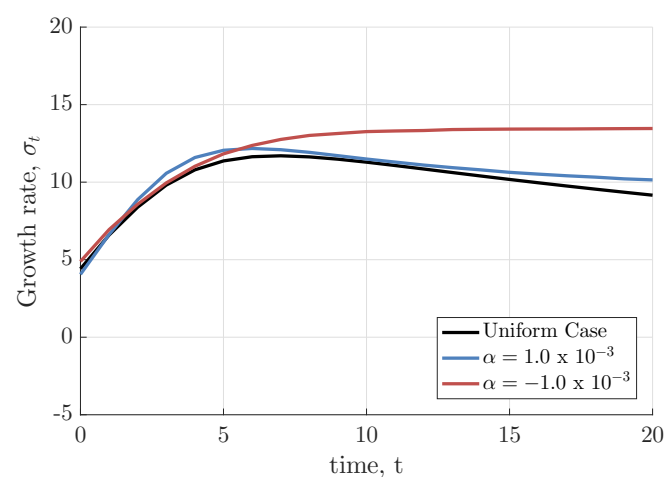

(b) $C a_{g}=1000$

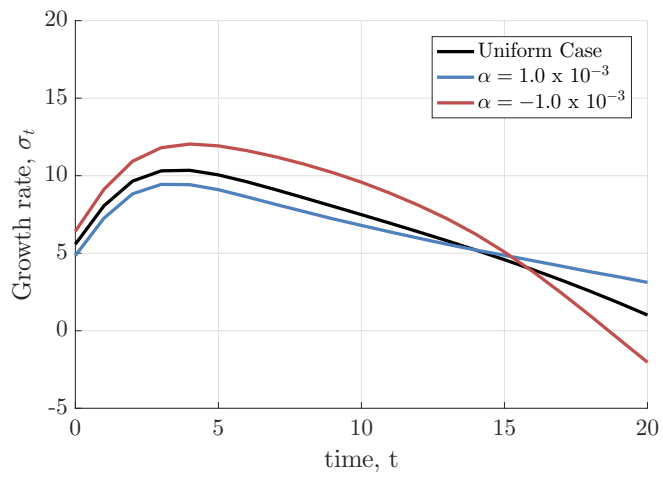

(c) $C a_{g}=2500$

Figure 8: Plots showing the non-linear growth rates with time for uniform, converging and diverging cells. $\beta=10$

Figure 8 shows that the converging cell has a smaller growth rate than the uniform cell at $C a_{g}=500$, supporting the stabilising mechanism of the transverse curvature highlighted by Figure 6 d. Increasing the capillary number to $C a_{g}=1000$, the growth rate increases beyond that of the uniform cell, as the front becomes more unstable. Interestingly, the diverging case at $C a_{g}=$ 1000 also exhibits a very slightly higher growth rate than the uniform, with remnants from the destabilising mechanism of the transverse curvature still apparent. In this transition regime, the growth rates show similar profiles due to the combined magnitude of the aperture effects. Prediction of the relative stabilisation compared to the uniform case in the transition regime therefore becomes very difficult in the full non-linear state.

At the highest capillary number of $C a_{g}=2500$ in Figure 8, the cases all show post stability transition properties, whereby the converging case has a higher growth rate than the uniform, and the diverging case has a smaller growth rate than the uniform. This relationship holds until $t \approx 14$ in the Figure, at which point bifurcation starts to occur. Bifurcation here refers to points along the 
interface where the curvature sign changes between time steps, and the normal velocity is reduced significantly in comparison to an unperturbed interface. After bifurcation at $t \approx 14$ in Figure 8 , the growth rate of the converging case drops below that of the uniform case, with the reverse true of the diverging case.

The low capillary number results in converging cases in the figures above show similar stabilising attributes to those presented by [11], in which air is injected into wetting mineral oil, with similar fluid properties to the high mobility ratio case presented here. They inject with a constant volumetric injection into a gradually converging rectilinear cell. As is the case with our converging cell, at low capillary numbers, [11] find that the interface can be almost completely stabilised to form a flat front.

Further work by [8] formulates the analytical growth rate for a circular fluid-fluid interface subject to an initial perturbation $\epsilon_{0}$ with a constant radial injection flux and a constant radial gradient in the cell separation. For this linear stability analysis to be valid at early times, the length scale of interface perturbation $r_{0} / n$ should be much smaller than the variation of the cell separation $\left|b_{0} / \alpha\right|$. The non-dimensional growth rate $\sigma_{a}$ is given by [8] as:

$$
\begin{gathered}
\sigma_{a}=-\left(\frac{\beta+1}{\beta}\right)\left(1+\frac{\alpha r_{0}}{b_{0}}\right)+\left(\frac{\beta-1}{\beta}+\frac{2 \alpha+(\pi / 4) b_{0}^{2} / r_{0}^{2}}{C a_{l}}\right) n-\frac{(\pi / 4) b_{0}^{2} / r_{0}^{2}}{C a_{l}} n^{3} \\
C a_{l}=\frac{12 \mu_{2} Q U_{n}\left(r_{0}\right)}{\gamma r_{0}}
\end{gathered}
$$

In equations $(35)$ and $(36), U_{n}\left(r_{0}\right)$ refers to the non-dimensional normal interface velocity at a radial location $r_{0}$ with corresponding plate separation $b_{0} . n$ is the non-dimensional wavenumber of the perturbation ( 8 in the current work). The parameter $Q / r_{0}$ in (36) appears due to the nondimensional velocity $U_{n}\left(r_{0}\right)$. Note we have also used the mobility ratio $\beta$ instead of the viscosity ratio originally used by [8] and we have included a correction of $\pi / 4$ to be consistent with the Young-Laplace matching condition used in this work.

To compare the early time frame linear stability of the numerical system with that given by the equation (35), we form a linear numerical growth rate $\sigma_{i}$ :

$$
\sigma_{i}=\left(\frac{U_{n}\left(r_{0}+\epsilon\right)-U_{n}\left(r_{0}\right)}{\epsilon_{0} U_{n}\left(r_{0}\right)}\right)\left(\frac{\beta+1}{\beta}\right)
$$

The linear numerical growth rate in (37) represents the growth rate of the interface at the perturbed initial state minus the growth rate at the unperturbed initial state. This growth rate differs from (34) in that it subtracts the non-linear velocity of the unperturbed interface as opposed to the linear velocity in (34). The non-linear growth rate includes a component due to the changing geometry, meaning at small initial amplitudes (37) gives a linearised approximation to the growth rate. Using corresponding scalings $\epsilon_{0}, \beta$ and $U_{n}\left(r_{0}\right)$, the linear numerical growth rate is comparable to the analytical linear stability given in (35) at small initial perturbations $\epsilon_{0}$ and at early time stages.

The linear analytical and numerical growth rates have been plotted for two different perturbation amplitudes in Figure 9. We plot the growth rate against the global capillary number, rather than the local capillary number in (35) to be consistent with our numerical simulations. The local capillary number in (35) can be found from the appropriate initial values, in order to work out the growth rate for a specific global capillary number.

In Figure 9 it can be seen that the numerical growth rate at the initial state shows very good agreement with that given by the analytical rate in (35). As the perturbation value is lowered to $\epsilon=0.01$ the numerical and analytical values become closer, as the numerical growth rate becomes more linear and closer to the approximation given by the LSA.

The initial state growth rates can be used to give insight into the front evolution when the capillary number is low. For the $C a_{g}=500$ cases, the growth rate is negative when the plates are converging in Figure 9, meaning the interface will be stable to perturbations of wave number, $n$. This is clearly demonstrated in Figure $6 \mathrm{a}$ and $6 \mathrm{~d}$, where the interfaces are almost completely stable. The reverse is also true of the uniform cell, in which the growth rate is positive, and clearly defined fingers form. 


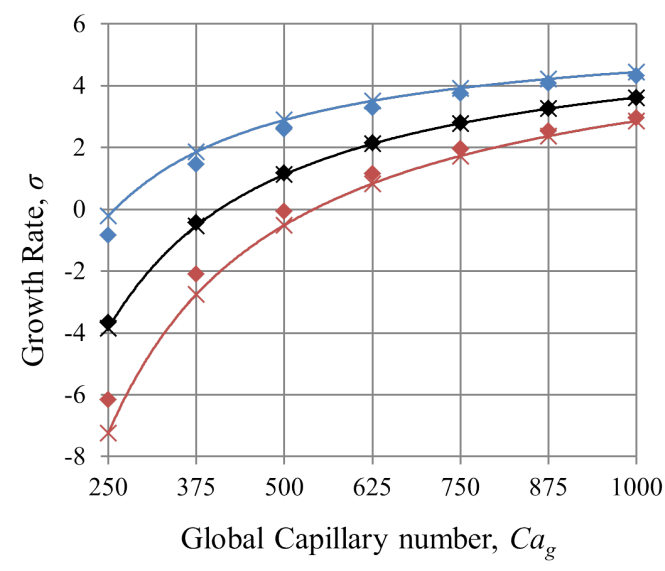

(a) $\epsilon_{0}=0.01$

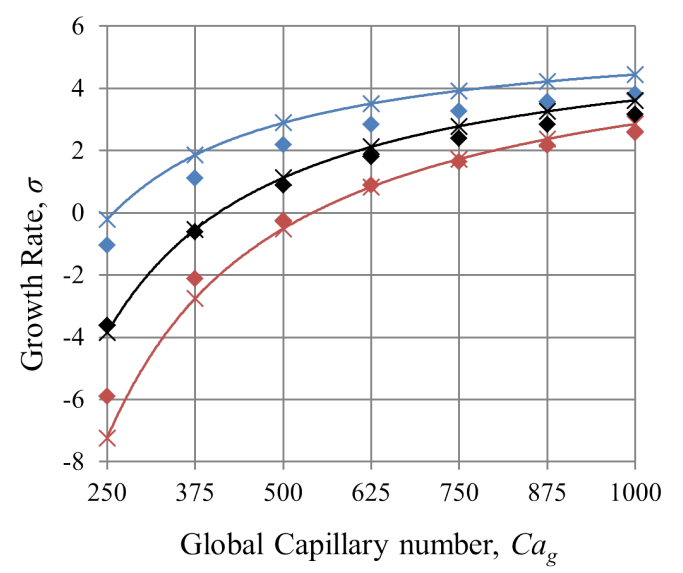

(b) $\epsilon_{0}=0.05$

Figure 9: Plots showing the analytical and numerical linear growth rates for different initial perturbations $\epsilon_{0}$ at the initial state. $\beta=10$. Solid lines and crosses represent the analytical growth rate $\left(\sigma_{a}\right)$, diamonds represent the numerical growth rate $\left(\sigma_{i}\right)$. — Uniform cell, — Converging cell, — Diverging Cell

In Figure 9, the initial state growth rates of the converging cases are always below that of the uniform cases, i.e. they are always more stable in the range $250 \leq C a_{g} \leq 1000$, with the reverse true for the diverging cases. However, in the non-linear simulations with $C a_{g}=1000$ at $t=24$ in Figure 6e, the front is further displaced in the converging case compared to the uniform case, indicating a higher growth rate. The initial state linear stability therefore cannot accurately predict the relative stability transition at high capillary numbers. Although the converging and uniform curves in Figure 9 will eventually cross and transition, this does not occur until much higher capillary numbers. Equation (35) can be used to find the relative stability transition point, by setting the growth rate with $\alpha=0$ equal to the growth rate when $\alpha \neq 0$. After rearrangement, the local and global critical capillary numbers for transition, $C a_{l t}$ and $C a_{g t}$ respectively, are given by:

$$
C a_{l t}=\frac{2 n}{1+\gamma}\left(\frac{b_{0}}{r_{0}}\right), \quad C a_{g t}=\frac{4 \pi n}{1+\gamma}\left(\frac{r_{0}}{b_{0}}\right)
$$

The above transition capillary numbers are therefore independent of the slope of the cell $\alpha$, and the initial perturbation $\epsilon_{0}$. For the values in the previous simulations, $C a_{l t}=0.14545$ and $C a_{g t}=9139.2$. The critical capillary numbers for transition are largely over predicted using the LSA, with the simulation critical capillary number lying in the range $500<C a_{g t}<2500$. Using a critical capillary number for transition based on the LSA provides an upper limit for which the capillary number must reach before the regime transitions to viscous dominated.

The over prediction of $C a_{g t}$ from the LSA and the numerical linear growth rate is mainly due to the relatively small contribution from viscous effects in comparison to capillary effects as $\epsilon_{0}$ reduces. The over prediction is best understood by considering the numerical linear growth rate. Here, since $U_{n}\left(r_{0}+\epsilon\right)$ and $U_{n}\left(r_{0}\right)$ both include the perturbed pressure induced from the varying plate separation (aperture effect 3), the final growth rate $\sigma_{i}$ only has a small, linear contribution from the perturbed pressure as $\epsilon_{0}$ gets smaller. However, the capillary contribution from the transverse curvature only features in $U_{n}\left(r_{0}+\epsilon\right)$, since $U_{n}\left(r_{0}\right)$ comes from a circular interface with constant $b(r)$. The final growth rate $\sigma_{i}$ therefore has a larger contribution from the transverse curvature than the perturbed pressure, meaning the transition to a viscous regime occurs much later than in the non-linear simulations.

The over prediction of $C a_{g t}$ using the linear approximations is also due to the fact that they are taken at the initial state with $\epsilon_{0}$. In the simulation setup, the ratio of the length scale of interface perturbation to the variation of the cell separation is $\left|\alpha r_{0} / n b_{0}\right|=0.013$, which is is below the initial perturbation amplitude of $\epsilon_{0}=0.05$, showing the initial non-linearity of the system. The relatively large initial perturbation also quickly evolves, with non-linear effects becoming more prominent and the effective $\epsilon$ increasing, meaning that the early time stage growth can be significantly different 
to the late stage growth. To better predict the transition in relative stability, we therefore turn to the non-linear growth rate, discussed in the next section.

\subsubsection{The effect of $\epsilon_{0}$ and $\alpha$ on the transition in relative stability}

To analyse the dependence of $C a_{g t}$ on $\epsilon_{0}$ and $\alpha$, the non-linear numerical growth rate is plotted at the initial state for various values of $\alpha$ and $\epsilon_{0}$ in Figure 10. There exists a clearly defined point in each plot where the relative stability transitions, which is independent of $\alpha$. This is expected from the LSA value of $C a_{g t}$, which does not include any contribution from $\alpha$, since at this point the capillary and viscous contributions are equal regardless of the slope of the cell.

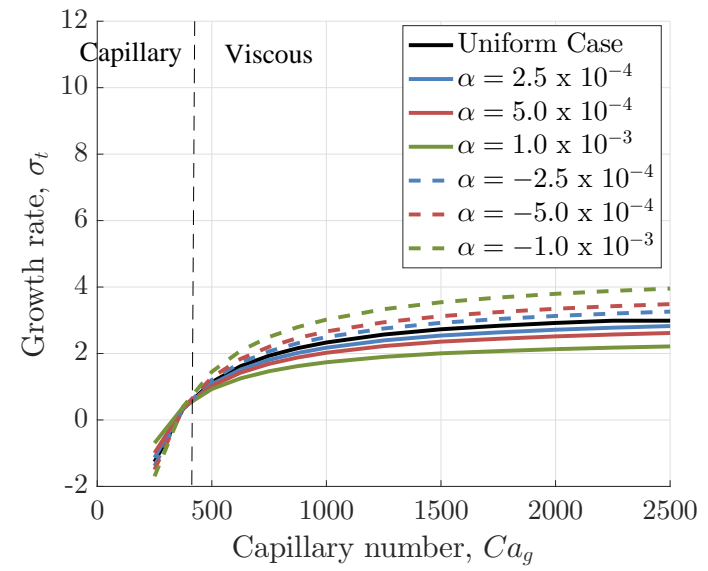

(a) $\epsilon_{0}=0.025$

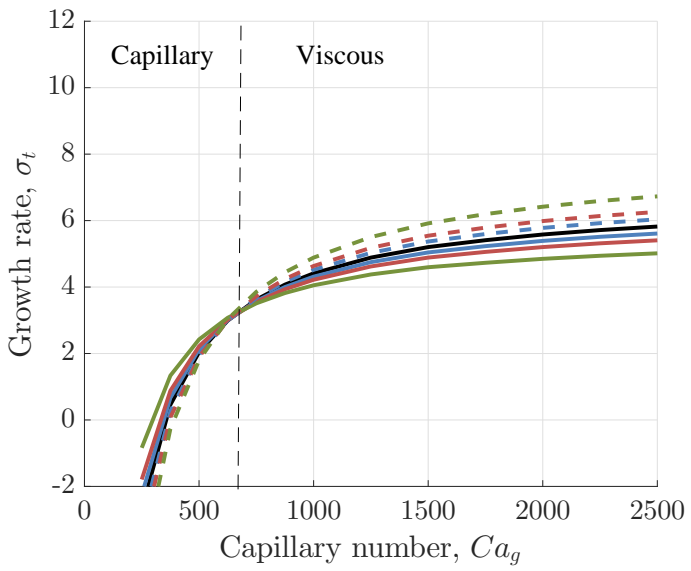

(b) $\epsilon_{0}=0.050$

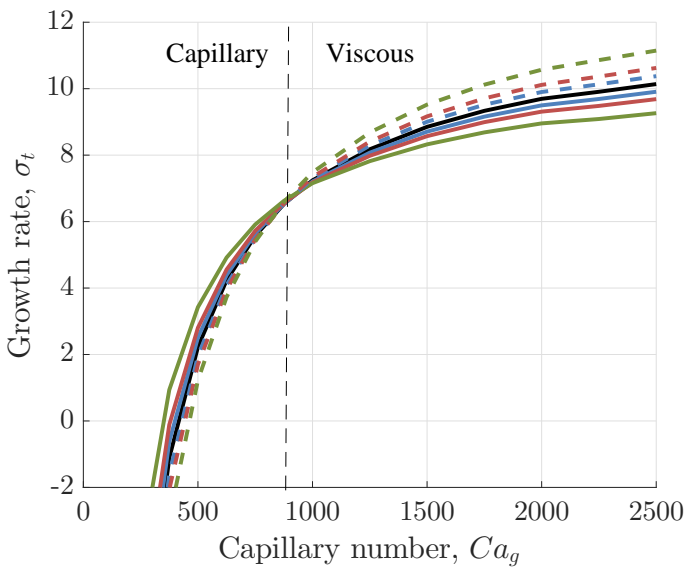

(c) $\epsilon_{0}=0.100$

Figure 10: Plots showing the initial state non-linear growth rates in cells with different $\alpha$ and $\epsilon, \beta=10$. The black dashed line marks the transition point from a capillary dominated to viscous dominated regime. The legend for each plot is shown in (a).

Interestingly, when $\epsilon_{0}$ is decreased in Figure 10, the apparent transition point also decreases. This appears at first counter intuitive, since it would be expected that as $\epsilon_{0}$ reduces, the transition point would increase towards that given by the LSA, i.e. $C a_{g t}=9139.2$. However, the disparity is due to the functional form of the non-linear growth rate at the initial state, which has a larger contribution from viscous effects compared to the linear growth rate. In the non-linear growth rate, the perturbed pressure is only included in the $U_{n}(r, t)$ term, meaning as $\epsilon_{0}$ decreases the growth rate comes almost entirely from the perturbed pressure (since the transverse curvature approaches a constant). Therefore, the initial state gives a smaller $C a_{g t}$ when $\epsilon_{0}$ is reduced, as viscous effects dominate in the early stages of the simulation. This result, although counter intuitive, is physical, since in the limit of $\epsilon_{0}=0$ there will be no transverse curvature effects, and the regime will be entirely viscous dominated. 
Although the above result is physical, it does not indicate the actual transition in relative stability seen in the non-linear simulations (nor does the linear $C a_{g t}$ prediction), since the initial perturbations grow with time and quickly reach a size where capillary effects become prominent. The question is now raised as to the transient behavior of the system, and if there is a point in the simulations at which perturbations of any starting $\epsilon_{0}$ have reached a size at which the transition in stability can be accurately predicted. We address this point by considering the difference in non-linear growth rates between the fronts in diverging and converging cells, i.e.:

$$
\Delta \sigma_{t}=\sigma_{t, \text { div }}-\sigma_{t, \text { conv }}
$$

where $\sigma_{t, \text { div }}$ and $\sigma_{t, \text { conv }}$ are the growth rates of the finger fronts in a diverging cell and converging cell respectively, with the same magnitude of $\alpha$. Negative values of $\Delta \sigma_{t}$ indicate a viscous dominated regime, whereby the interface in the converging cell is displacing quicker than the interface in the diverging cell, due to the relatively large contribution from the perturbed pressure over the transverse curvature. Positive values of $\Delta \sigma_{t}$ indicate a capillary dominated regime.

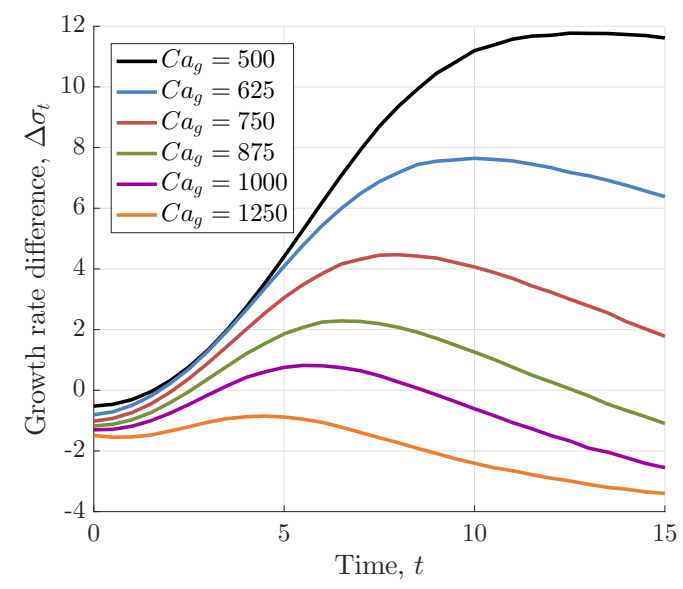

(a) $\epsilon_{0}=0.025$

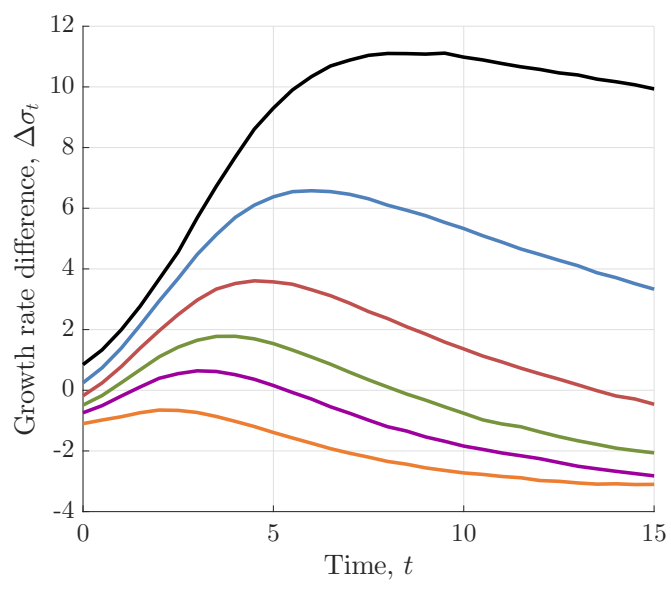

(b) $\epsilon_{0}=0.050$

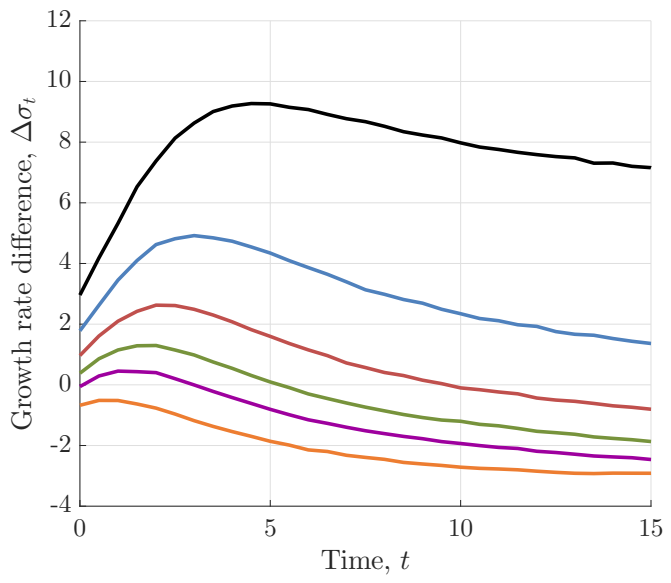

(c) $\epsilon_{0}=0.100$

Figure 11: Plots showing the difference in growth rates between diverging and converging cells with time, for different $\epsilon_{0} \cdot|\alpha|=0.001$. The legend for each plot is shown in (a).

In Figure 11, $\Delta \sigma_{t}$ has been plotted for various $C a_{g}$ at different $\epsilon_{0}$ values. From this, it can be seen how some simulations (particular at $\epsilon_{0}=0.025$ ) start in a viscous dominated regime, i.e. $\Delta \sigma_{t}<0$, but change to a capillary dominated regime later in time once the perturbation has grown. This implies that the transition in relative stability is both dependent on the capillary number and time. Assessing the growth rates from the simulation at different points in time can 
yield entirely different results for the capillary number at which the relative stability transitions, i.e. in Figure 11(b) at $t=0,625<C a_{g t}<750$, whereas at $t=5,1000<C a_{g t}<1250$.

Due to the transient issues above, we define the transition in relative stability as the capillary number $C a_{g t}$ at which $\Delta \sigma_{t}<0$ for all time in the simulation up until the first bifurcation. This statement requires that the interfacial displacement is always viscous dominated, and the growth rate in the converging case should always be larger than the corresponding converging case, up until bifurcation occurs. This transition point is therefore an upper bound measure of when the regime is in the viscous limit, in which it always exhibits post-transition aperture effects.

In Figure 11, the capillary number at which the transition in relative stability occurs is in the range $1000<C a_{g t}<1250$. This range is independent of $\epsilon_{0}$, since $\epsilon_{0}$ only changes the absolute magnitude of $\Delta \sigma_{t}$ and not the relative spacing between the $C a_{g}$ curves in Figure 11. The transition in relative stability will therefore occur indefinitely for any initial perturbation, with $\epsilon_{0}$ only controlling the time it takes for the transition to become apparent in the system. For very small $\epsilon_{0}$, the initial perturbation starts in the viscous regime for all but the very smallest capillary numbers, meaning the transition in relative stability can take a long time to become apparent in the system, but will occur at the same capillary number as larger values of $\epsilon_{0}$ given enough time.

From this analysis, it can be concluded that the initial growth rate (whether linear or nonlinear) cannot be effectively used to predict the transition in relative stability, since the interplay between viscous and capillary effects with the growing perturbation can change dramatically with time. Instead, the relative stability must be assessed at all times up until the first bifurcation, with the condition that $\Delta \sigma_{t}<0$ defining the capillary number for transition. $C a_{g t}$ is independent of both $\alpha$ and $\epsilon_{0}$ as predicted by the LSA, with only the timescale of events altered by $\epsilon_{0}$.

The results presented in this section link the linear stability analysis works of $[11,8]$ and the experimental results of [9], detailing the explicit mechanisms that cause the transition in relative stability when the capillary number is increased. Although the linear stability analysis gives a good prediction for the point at which absolute instability occurs and defined viscous fingers form, it cannot accurately predict the point at which the relative stability (compared to an interface in a uniform cell) transitions. By using the non-linear numerical growth rate, we can predict the critical capillary number at which a relatively stable interface becomes relatively unstable in comparison to the corresponding interface in a uniform cell. At lower capillary numbers, the flow is in a capillary limited regime, where gradients in the capillary pressure control the flow path and stabilisation can occur in converging geometries (e.g. the work of [11]). However at high capillary numbers , after the transition point, the flow is in a viscous limited regime and the permeability distribution controls the flow path meaning fingers can be sharpened and channeled in converging rectilinear geomtries (e.g. the work of [9]).

\subsection{Sharply converging/diverging geometry}

To further analyse low capillary number aperture effects on the fingering regime, we now focus on the fingering instability after defined fingers have formed. For this we use a uniform cell with a sharply varying section occurring far downstream of the initial interface position. We use a constant gradient variance from the same homogeneous separation as before, $\bar{b}=0.01$; however, the magnitude of the gradient is larger at $|\alpha|=0.0025$. The constant gradient section begins at $r=4$ and finishes at $r=5$, allowing the late stage exploration of secondary tip-splitting instabilities.

Before we proceed, it is worth discussing the validity of the lubrication approximation in HeleShaw cells when the plate separation varies sharply. We do this in order to provide some justification for the use of the model in the work presented here, whereby the spatial derivatives of plate separation can be quite large.

In the works of [24],[25] and [26], the validity of the lubrication approximation for fractures is discussed when the fractures are small and surface roughness may affect the flow regime. Under certain conditions, [26] finds that the difference in fluxes predicted between the lubrication and Stokes models can vary by a factor of two. [24] and [25] provide quantitative limits for the validity of the lubrication theory, based on the profile of the fractures. [24] find that the wavelength $\gamma_{h}$ of the aperture variation must exceed fifty times the standard deviation of the aperture height distribution $\sigma_{h}$ for the lubrication model to be valid. This ensures that the velocity gradients 
along the length of the fracture are much smaller than perpendicular to the fracture. [25] later refine this limit to only $5 \sigma_{h}$.

Applying the ratio $r_{l}=\gamma_{h} / \sigma_{h}$ to the cases here, the validity of the lubrication model can be assessed. The ratio of the wavelength of the variation of the plate separation to the standard deviation of the plate separation profile here is $r_{l}=1384$. This is much greater than the lowest limit of 5 given by [25], validating the use of the lubrication model in this work. The very large values here are due to the fact that although the spatial derivatives in the plate separation are quite large, the absolute change in the cell profile is small, meaning the velocity gradients in the plane of the cell will still be much smaller than those in the perpendicular direction.

Figure 12 shows the late stage interfacial evolution of the converging case for $C a_{g}=500$. Here, the rapid stabilisation of the interface causes it to form a very flat front, with the finger sides almost merging into one continual interface. The finger bases have remained entirely unaffected, as they exist in regions of uniform geometry and have almost stagnated.

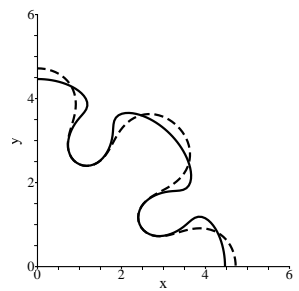

(a) $\mathrm{t}=45$

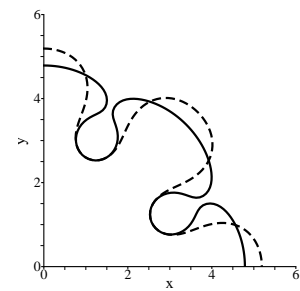

(b) $\mathrm{t}=55$

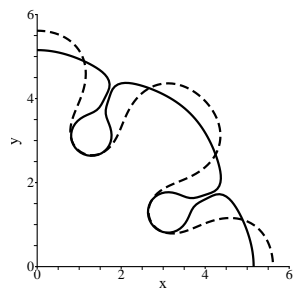

(c) $\mathrm{t}=65$

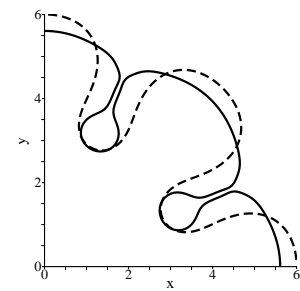

(d) $\mathrm{t}=75$

Figure 12: Time evolution plots for the converging cell case, $\alpha=-0.0025, C a_{g}=500$. - Converging cell, ---Uniform cell. $\beta=10$

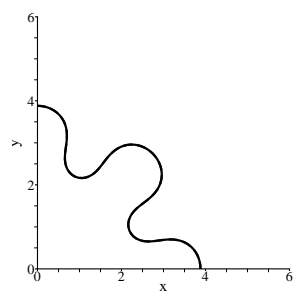

(a) $\mathrm{t}=30$

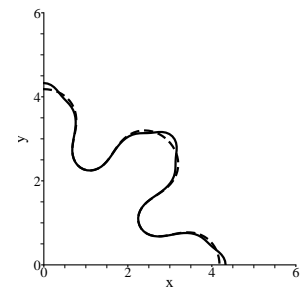

(b) $\mathrm{t}=35$

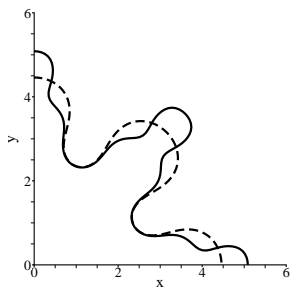

(c) $\mathrm{t}=40$

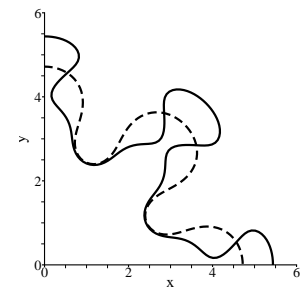

(d) $\mathrm{t}=45$

Figure 13: Time evolution plots for the diverging cell case, $\alpha=0.0025, C a=500$. — Diverging cell, ---- Uniform cell. $\beta=10$

Although the front is relatively flat and has been accelerated by the converging geometry in Figure 12, the stabilisation given by the increasing capillary pressure at the finger front keeps the interface from bifurcating into many smaller fingers. The fingers proceed to spread and expand under the stabilising aperture effect 1 of the converging geometry, with small lubrication layers forming between the fingers. Due to the complete immiscibility of the fluids in these simulations, there will always exist a small layer of the outer fluid separating the advancing fingers, meaning that droplets of the external fluid inside the moving plume never completely form. The lubrication layer formed in Figure 12 is fully resolvable with both the BEM and RBF-FC methods, in which several overlapping local systems exist within the layer itself.

In the diverging geometry case in Figure 13, the front is rapidly destabilised as it enters a region of very large plate separation. This causes a sudden decrease in capillary pressure, meaning it is much easier for the interface to displace in this region. The interface quickly forms a throat of very small radius at the entrance to the diverging section at $r=4$, which becomes thinner and thinner with time. After $t=45$, the throat continues to shrink until it reaches a stage where it is so thin that it collapses and the finger downstream of the throat detaches to form a separate bubble. The simulation ends before the breaking effect, as the throat size is too small to fit sufficient nodal points inside to form suitable local systems in the RBF-FC method. Also, the model cannot accurately predict the bubble detachment and surface tension snapping effect. However, the model 
can predict up until a time very close to the 'breaking', where it can be fairly accurately predicted that the throat will collapse and the finger will detach. After this, the same process will likely occur again, as the remaining interface that forms the main plume will enter the expanding region, where the same destabilising effects would be felt. In this way, we infer that the expansion acts as a barrier breaking the plume into a succession of droplets that will be convected by the displaced fluid.

Physically, a sudden change in cell geometry is analogous to a sharp change in porous medium permeability. For the diverging case, the interface effectively travels from a region of low permeability (such as sandstone), to a region of very large permeability (such as fractured shale). The periodic shedding of the interface would be highly desirable in $\mathrm{CO}_{2}$ sequestration, as the interface surface area would be effectively increased, meaning the rate of dissolution and total amount of dissolution trapping would increase significantly. For the converging case, the interface hits a permeability barrier, where the permeability decreases rapidly. This could represent an area of porous medium under greater compression than at the origin of the injection, where the interface would stabilise and the amount of trapping would decrease.

\subsection{Periodic Hele-Shaw cells}

In this final results section, we analyse immiscible displacement in Hele-Shaw cells with significant heterogeneities and anisotropy in the cell permeability, in order to demonstrate the different flow features that can occur when the permeability variation is not necessarily aligned with the flow direction. Up until this point, the maximum gradient in plate separation (and permeability) has been aligned with the direction of displacement of the fingers, as in previous rectilinear and radial works $[8,9,11]$. This is useful to derive the key mechanisms behind the stability of the viscous fingers and the associated transition in relative stability, in geometries that are easy to replicate both numerically and experimentally. However, in real porous media, the variation in permeability will not be aligned with the direction of displacement, and will vary anistropically in the domain, which can lead to significant variation from the displacements in homogeneous media.

Using a periodic function for the plate separation and cell permeability, viscous fingering can be analysed in geometry that more closely resembles the natural variations found in aquifer permeabilities. We use this section to discuss fluid trapping and preferential flow mechanisms that occur due to permeability heterogeneity, and the relative magnitude of the aperture effects with more localised variations in permeability. For brevity we do not present the analytical functions used to define the plate separations, instead showing contour plots of the associated variations, noting that in all cases $\bar{b}=0.01$

We present results from a Hele-Shaw cell with a plate separation that varies periodically in both Cartesian coordinates. The same eight finger starting interface is used as in previous simulations, however, the plate separation now exhibits quarter-fold symmetry. The separation variation contour is shown in the background of Figure 15. In Figure 14, interface plots for the periodic cells and corresponding uniform cells can be seen at $t=40$ for different capillary number regimes. In Figure 15 we see the transient evolution of the interfaces, overlaying contour plots of the plate separation.

In the low capillary number regime in Figures 14a and 15a, the interfacial displacement has been completely controlled by aperture effect 1 . The interface bears little resemblance to the uniform case, with local gradients in capillary pressure due to the changing transverse curvature altering the flow path significantly. The initial stabilisation of the interface due to locally converging sections (and hence positive capillary pressure gradients) leads to an almost circular interface. However, shortly afterwards, small fingers are formed from the residual perturbations that extend into the domain. This presents an example of flow at the capillary limit, where the capillary pressure gradient (aperture effect 1) has significantly re-distributed the fluid from its original flow path, with the heterogeneity defining the distribution of fluid.

In the higher capillary number regime in Figures $14 \mathrm{~b}$ and $15 \mathrm{~b}$, we see a completely different flow regime that is viscous limited. The finger evolving in the periodic cell at $45^{\circ}$ to the horizontal has a completely different bifurcation mode to the fingers travelling parallel to the $\mathrm{x}$ - and $\mathrm{y}$-axes in 14b. The fingers travelling parallel to the axes have been sharpened significantly due to continued acceleration of the finger tips in an anistropic medium, whereas the $45^{\circ}$ finger evolves in a very 
similar manner to the uniform case due to the relatively homogeneous permeability along the direction of travel.

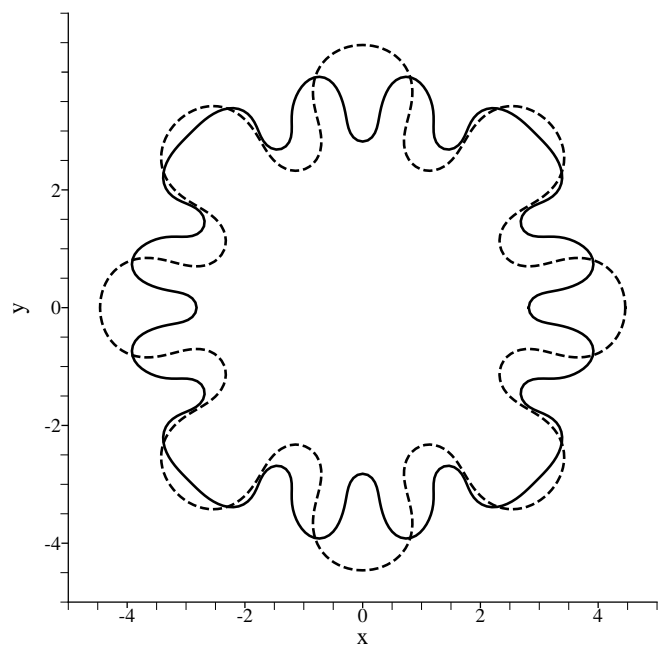

(a) $C a_{g}=500$

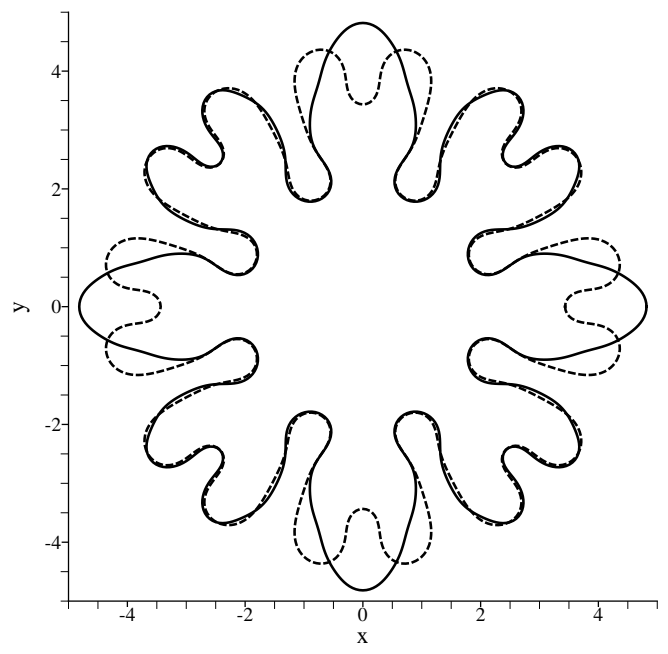

(b) $C a_{g}=2500$

Figure 14: Interface plots at $t=40$ for the Cartesian periodic cell and uniform cell. — Cartesian periodic cell,- - Uniform cell

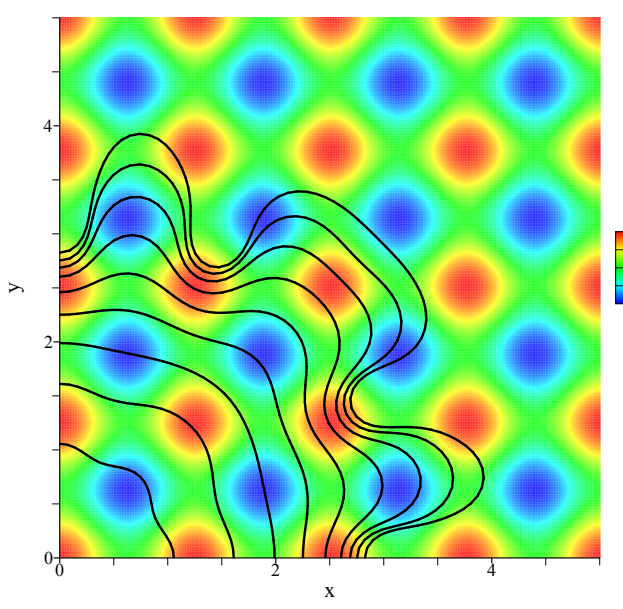

(a) $C a_{g}=500$

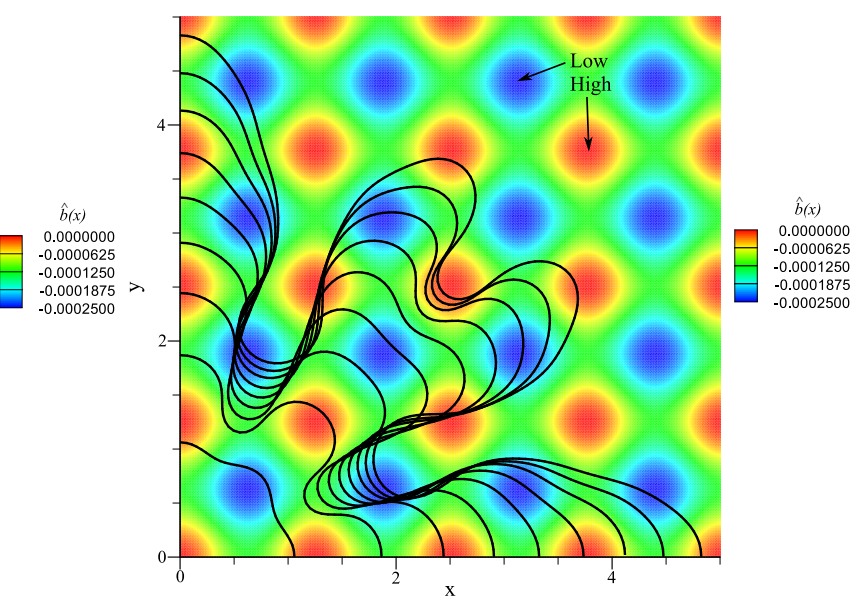

(b) $C a_{g}=2500$

Figure 15: Interface plots at $\Delta t=5$ increments from $t=0$ to $t=40$ for the Cartesian periodic case. The interfaces overlay a contour plot of the plate separation variation, $\hat{b}(x)$.

Due to the large capillary number in this case, the transverse curvature and capillary pressure gradient (aperture effect 1) have very little effect on the overall finger evolution. The fingers parallel to the axes generally have their tips in regions of higher permeability (and hence mobility) compared to the sides of finger fronts as the interface displaces, which aperture effect 2 causes the finger sharpening. As the overall change in plates separation is small, the change in pressure gradient (aperture effect 3) is relatively small. Here, although the plate separation and permeability do not vary in magnitude significantly, they do vary anisotropically, meaning in the viscous limit the flow can be directed down the path aligned with high permeability.

The sharpening behaviour is similar in nature to that in the rectilinear case presented by [9]. In [9], a gradually converging rectilinear cell was found to sharpen the fingers, as the tip would be accelerated more significantly than the sides of the finger fronts. The pressure gradient at the finger tip would be increased locally, with aperture effect 3 causing the increased tip velocity (the 
mobility variation is small around the finger front). However, in the present case, a locally high mobility (aperture effect 2) causes the increased velocity. The result in both cases is that the finger tip experiences a higher velocity than the sides of the finger front, and the finger is sharpened. This result is in contrast to what has been seen in previous sections using radially symmetric cases, as the finger tip and the sides of the finger front exist in areas of significantly different mobility and spatial variation in mobility.

The result presented in Figure 15 shows the difference in finger morphologies when the plate separation and permeability vary non-uniformly with respect to the flow direction. Although the case presented is for radial Hele-Shaw flow in a periodic cell, the features clearly highlight the difference between high capillary number (viscous limit) Hele-Shaw flow in gradually converging radial and rectilinear cells. For radial cells, the acceleration of the flow through a constant gradient converging geometry flattens the front and enhances bifurcation, as the finger fronts and sides both displace into equally converging flow paths. However, in rectilinear cells with a constant gradient converging geometry, the front is sharpened as the finger tip displaces into a more sharply converging path than the sides of the finger fronts.

These final results demonstrate the effect of local variations in cell plate separation (and cell permeability). This can also be viewed in the sense of aquifer permeability, with large permeability changes such as cracks and fractures resulting in highly directional flow paths. In the results presented here, small changes in permeability result in largely the same interfacial displacement if the capillary number is high enough, unless the permeability distribution is highly anisotropic. This infers that in the $\mathrm{CO}_{2}$ injection cases, small variations in aquifer permeability across the whole plume are unlikely to alter the resulting displacement regime in the early stages of injection when the capillary number is high, unless there are faults or features with drastically differing permeabilities present. However, when the capillary number is very small (capillary limit), the finger displacement can change significantly, and is completely controlled by local capillary pressure gradients.

\section{Conclusion}

In this work, we investigated the stability of immiscible viscous fingering in Hele-Shaw cells with spatially varying permeability, comparing the interfacial displacements and growth rates with corresponding cases in cells with uniform permeability. We examined the onset of fingering and late stage tip splitting instabilities for different cell geometries across a wide range of capillary numbers, demonstrating the transition in fingering mechanisms. Numerical experiments with gradually converging/diverging Hele-Shaw cells highlighted three aperture effects that controlled the interfacial stability, for regions of locally decreasing (converging) permeability these were:

1. Stabilisation through an increased transverse curvature (positive capillary pressure gradient).

2. Deceleration due to a reduced fluid mobility.

3. Acceleration due to an increased (viscous) pressure gradient.

In regions of locally increasing (diverging) permeability, the effects were reversed. At low capillary numbers, aperture effect 1 and 2 were found to dominate, stabilising the interface in converging cases and destabilising in diverging cases (when compared to interfaces in uniform cells). At higher capillary numbers, the relative stability transitioned, and converging cells destabilised the interface in comparison to uniform cells, with diverging cases being relatively more stable.

The transition in relative stability was found using the transient, non-linear numerical growth rate to be in the range $1000<C a_{g t}<1250$, independent of both $\alpha$ and $\epsilon_{0}$, providing a better accordance with the numerical results than the initial state growth rate. These results using simple Hele-Shaw geometry link the previous works performed at either a low or high capillary number, providing a detailed analysis of the mechanisms behind the displacement and the relative stability transition.

Further analysis on more anisotropic Hele-Shaw cells revealed the effects of local variations in permeability and fluid re-distribution that could occur. Below the relative stability transition point, the flow can be re-distributed by gradients in the capillary pressure. This created interfacial displacements that were far from the uniform cell case when the permeability distributions were 
periodic. When the capillary number was increased beyond the relative stability transition point, the flow becomes viscous dominated, meaning the flow regime is largely controlled by the underlying permeability distribution. If the permeability distribution is significantly anisotropic, the fluid can be directed through areas of high permeability in preference to low permeability regions, forming elongated fingers.

\section{Acknowledgements}

The authors would like to thank the University of Nottingham HPC team for the use of the Minerva supercomputer cluster. The present work has been supported by an EPSRC post-graduate research scholarship.

\section{References}

[1] P.G. Saffman and G.I. Taylor. The penetration of a fluid into a porous medium or Hele-Shaw cell containing a more viscous liquid. Proceedings of the Royal Society of London. Series A: Mathematical and Physical Sciences, 245(1242):312 - 329, 1958.

[2] G. Lovoll, Y. Meheust, K.J. Maloy, E. Aker, and J. Schmittbuhl. Competition of gravity, capillary and viscous forces during drainage in a two-dimensional porous medium, a pore scale study. Energy, 30:861 - 872, 2005.

[3] R. Lenormand, E. Touboul, and C. Zarcone. Numerical models and experiments on immiscible displacements in porous media. Journal of Fluids Mechanics, 189:165 - 187, 1988.

[4] J. Bear and A.H.-D. Cheng. Modeling Groundwater Flow and Contaminant Transport (Theory and Applications of Transport in Porous Media). Springer Netherlands, 2010.

[5] K. Pruess and N. Müller. Formation dry-out from $\mathrm{CO}_{2}$ injection into saline aquifers: 1. Effects of solids precipitation and their mitigation. Water Resources Research, 45(W03402), 2009.

[6] B. Lamy-Chappuis, D. Angus, Q. Fisher, C. Grattoni, and B.W.D. Yardley. Rapid porosity and permeability changes of calcareous sandstone due to $\mathrm{CO}_{2}$ - enriched brine injection. Geophysical Research Letters, 41:399 - 406, 2014.

[7] D.C. Thomas and S.M. Benson. Carbon Dioxide Capture for Storage in Deep Geologic Formations - Results from the $\mathrm{CO}_{2}$ Capture Project: Vol 2 - Geologic Storage, chapter Prediciting and Monitoring Geomechanical Effects of $\mathrm{CO}_{2}$ injection, pages $751-766$. Elsevier, 2015.

[8] T.T. Al-Housseiny and H.A. Stone. Controlling viscous fingering in tapered Hele-Shaw cells. Physics of Fluids, 25(092102), 2013.

[9] H. Zhao, J. Casedemunt, C. Yeung, and J.V. Maher. Perturbing Hele-Shaw flow with a small gap gradient. Physical Review A, 45:2455 - 2460, 1992.

[10] E.O. Dias and J.A. Miranda. Finger tip behaviour in small gap gradient flows. Physical Review E, 82(056319), 2010.

[11] T.T. Al-Housseiny, P.A. Tsai, and H.A. Stone. Control of interfacial instabilties using flow geometry. Nature Physics, 8:747 - 750, 2012.

[12] T. Maxworthy. Experimental study of interface instability in a Hele-Shaw cell. Physical Review A, 39(11):5863 - 5866, 1989.

[13] T.T. Al-Housseiny, I.C. Christov, and H.A. Stone. Two-phase fluid displacement and interfacial instabilties under elastic membranes. Physical Review Letters, 111(034502), 2013.

[14] D. Pihler-Puzović, R. Périllat, M. Russell, A. Juel, and M. Heil. Modelling the supression of viscous fingering in elastic-walled Hele-Shaw cells. Journal of Fluid Mechanics, 731:162 - 183, 2013. 
[15] D. Pihler-Puzović, P. Illien, M. Heil, and A. Juel. Supression of complex finger patterns at the interface between air and a viscous fluid by elastic membranes. Physical Review Letters, 108(074502), 2012.

[16] C.W. Park and G.M. Homsy. Two phase displacement in Hele-Shaw cells: Theory. Journal of Fluid Mechanics, 139:291 - 308, 1984.

[17] E. Alvarez-Lacelle, J. Ortin, and J. Casademunt. Relevance of dynamic wetting in viscous fingering patterns. Physical Review E, 74(025302(R)), 2006.

[18] P.H. Anjos and J.A. Miranda. Radial viscous fingering: Wetting effects on pattern-forming mechanisms. Physical Review E, 88(053003), 2013.

[19] S.J. Jackson, D. Stevens, D. Giddings, and H. Power. Dynamic-wetting effects in finitemobility-ratio Hele-Shaw flow. Physical Review E, 92(023021), 2015.

[20] S.J. Jackson, D. R. Stevens, H. Power, and D. Giddings. A boundary element method for the solution of finite mobility ratio immiscible displacement in a Hele-Shaw cell. International Journal for Numerical Methods in Fluids, 78(9):521 - 551, 2015.

[21] S.J. Jackson, D. Stevens, D. Giddings, and H. Power. An adaptive RBF finite collocation approach to track transport processes across moving fronts. Computers and Mathematics with Applications, 71(1):278 - 300, 2016.

[22] D. Stevens, H. Power, C.Y. Meng, and D. Howard. An alternative local collocation strategy for high-convergence meshless PDE solutions, using radial basis functions. Journal of Computational Physics, 245:52 - 75, 2013.

[23] D. Stevens, H. Power, M. Lees, and H. Morvan. A local hermitian RBF meshless numerical method for the solution of multi-zone problems. Numerical Methods for Partial Differential Equations, 27(5):1201 - 1230, 2011.

[24] S.R. Brown. Fluid flow through rock joints: The effect of surface roughness. Journal of Geophysical Research, 92(B2):1337 - 1347, 1987.

[25] R.W. Zimmerman, S. Kumar, and G.S. Bodvarsson. Lubrication theory analysis of the permeability of rough-walled fractures. International Journal of Rock Mechanics and Mining Sciences \& Geomechanics Abstracts, 28(4):325 - 331, 1991.

[26] V. Mourzenko, J.-F. Thovert, and P. Adler. Permeability of a single fracture; validity of the Reynolds equation. Journal de Physique II, EDP Sciences, 5(3):465 - 482, 1995. 